\title{
LE SON DANS LA BANDE DESSINÉE - RÉPRÉSENTATION ET IMAGINATION DE LA DIMENSION AUDITIVE
}

Marco Pellitteri ${ }^{*}$

Résumé: Cet essai offre une perspective générale des différentes manières dont la visualisation des sons peut se produire dans les récits de bd. Il discute des caractéristiques principales de la façon dont l'action combinée de signes visuels et de récits efficaces peuvent encourager l'imagination sonore des lecteurs à créer ou à recréer dans leur esprit des versions personnelles de sons censés être une partie vivante de l'expérience narrative.

Mots clé: Son. Onomatopées. Lettrage.

\section{INTRODUCTION}

L es bandes dessinées (BD) sont généralement conçues comme un médium purement visuel. Cependant, la plupart d'entre elles présentent également des symboles graphiques évoquant des sensations et des situations liées aux quatre autres sens traditionnels. L'audition est le deuxième sens physiologique, par ordre d'importance, utilisé lors de la création et de la lecture de $\mathrm{BD}$. Les bandes dessinées présentent, ou peuvent présenter, de nombreux signes et symboles visuels d'ordre sémiotique qui se réfèrent aux sons et aux bruits. Il y a, en outre, d'autres manières - plus subtilement narratives tout en restant intrinsèquement visuelles - par lesquelles les créateurs de BD suggèrent aux lecteurs que certains phénomènes auditifs se produisent dans l'histoire.

Il y a deux façons d'exprimer la multi-sensorialité dans les BD. La première se rapporte à la diégèse: à ce qui se passe dans l'histoire et à la façon dont les dessins, les conventions graphiques et la narration transmettent des déclencheurs visuels et/ou narratifs pour évoquer chez le lecteur des sensations internes ou des expériences passées. La seconde fait référence à la matérialité de la BD. Ian 
Hague $(2012,2013)$ a adopté cette deuxième approche, mais je choisis de l'écarter ici, convaincu que la première approche présente les implications multisensorielles les plus utiles à l'expression des idées que je développe ci-après ${ }^{1}$.

Établir qu'un objet physique est lié aux sens va de soi. À l'inverse, établir une multidimensionnalité polysensorielle de la BD du point de vue diégétique et graphique déconstruit l'idée selon laquelle la BD est un médium uniquement "visuel ". Le pouvoir narratif de la BD consiste dans la capacité à suggérer, dans les mouvements mentaux du lecteur, des accélérations et des ralentissements liés au temps, des odeurs et des saveurs, des sensations tactiles. Cette capacité existe même à travers la technologie maigre de la page imprimée, qui héberge, au contraire, un médium riche et polyvalent.

\section{UNE FORME MULTI-MÉdIALE, MULTI-MODALE ET MULTI-SENSORIELLE}

Scott McCloud (1993, 2000, 2006), auteur de BD qui a expliqué ses opinions sur cette forme dans une trilogie d'œuvres, a affirmé que les BD sont un "support mono-sensoriel " (MCCLOUD, 1993), soulignant l'aspect visuel. En revanche, les chercheurs spécialistes des médias qui étudient les BD ont convergé sur la manière dont celles-ci stimulent ou suggèrent une plus grande sensorialité (PELLITTERI, 1998, 2007; SABIN, 2000, p. 52; HAGUE, 2012, 2013).

Ici comme dans mes études précédentes, je présente et théorise la BD comme un médium de masse, une forme d'art populaire et d'expression culturelle et une forme de narration. Ces trois niveaux de signification et structuration, imbriqués l'un dans l'autre, sont toujours présents dans les BD, mais à des degrés divers. En lien direct avec les suggestions sonores apportées par la conception graphique, les codes visuo-symboliques et la narration typique de ce médium, les BD relèvent pleinement du domaine de ce que Don Ihde a étudié comme étant "l'imagination auditive " (IHDE, 2003, p. 61).

Les $\mathrm{BD}$ encouragent une lecture créative à travers les langages qui les composent. Ces langages sont multiples et forment ensemble un "super-langage " (PELLITTERI, 1998, 1999), un code mixte composé de plusieurs " co-langages ", qui sont une caractéristique majeure (un proprium) de la BD. Ce code composite (voir aussi, sur ce sujet, COHN, 2013) combine des éléments iconiques avec des processus d'interprétation plus articulés, tels que des séquences d'images exploitant des modalités de narration visuelle que les BD ont en commun avec le cinéma - disparitions en fondu, narration en parallèle, montage intégré et ainsi de suite (LACASSIN, 1972); des éléments de mise en scène théâtrale; des styles provenant de formes antérieures de représentation comme la xylographie, l'illustration grotesque et la caricature politique; et une articulation des cases entre elles qui suivent des tailles, formes, distributions et hiérarchies variés et qui donnent au lecteur des rythmes et des informations de lecture différents. Tout cela engendre, dans l'esprit du lecteur, une forte implication à plusieurs niveaux.

En outre, comme le dit Charles Hatfield, ces niveaux peuvent être considérées comme un " art de la tension ": en parlant des pages de BD et des stimula-

\footnotetext{
Il y a certainement des situations tangentes, telles que (dans le domaine de l'imprimé) la possibilité de gadgets, d'instructions de lecture ou d'odeurs ajoutées; ou encore tels que (dans le domaine du numérique) des sons ou des animations. Mais tous ces artifices engendrent un glissement du médium bd vers autre chose: une " bd augmentée ", si I'on peut dire; en tous cas plus une bd au sens où on l'entend habituellement. Sur cette approche, outre Hague (2013). Voir aussi McCloud (2000).
} 
tions que le plaisir de lire déclenche chez leur lecteur, la page "fonctionne à la fois en tant que séquence et en tant qu'objet, à voir et à lire de manière linéaire autant que non linéaire, de manière holistique " (HATFIELD, 2005, p. 32).

\section{LES TROIS STRATES DE PERCEPTION DANS LES BANDES DESSINÉES}

Les strates en question sont les trois types de perception sensorielle: spatiale, temporelle et physiologique. Cette dernière, notamment, comprend: la vue, l'ouïe, l'olfaction, le goût et le toucher.

STRATE DE LA COMPRÉHENSION SPATIALE. La perception physico-spatiale, par le lecteur, des lieux/objets statiques ou dynamiques représentés dans les cases de BD n'est pas une question centrale dans notre discours sur la dimension auditive. Je ne traiterai donc pas de ce sujet (pour cela, voir LEFĖVRE, 2011; COOK, 2012). Je dois cependant au moins souligner que la façon dont les images sont présentées dans l'histoire d'une BD peut donner au lecteur des indications sur l'espace, les rapports spatiaux entre les objets, la perspective, l'orientation et, parfois même, du vertige (PELLITTERI, 1998, p. 21-87).

STRATE DE LA COMPRÉHENSION TEMPORELLE. En ce qui concerne la façon dont le temps est traité dans les BD (durée, vitesse, rythme, accélérations, ralentissements, pauses et bonds chronologiques), le story-telling ou récit, qui est l'ensemble des modalités de narration verbo-visuelle utilisées dans une histoire (MCCLOUD, 2006), peut sensiblement modifier la perception du flux temporel, non seulement dans l'histoire fictive, mais aussi à l'extérieur, dans le monde réel. C'est-à-dire que le lecteur peut percevoir différemment les cadences et les durées grâce aux variations d'équilibre dans le temps diégétique et dans le rythme de lecture que les créateurs conçoivent intentionnellement: par exemple, si dans une page de BD nous voyons une case beaucoup plus grande que les autres, cet élément nous incitera à nous y attarder plus longtemps que sur les autres cases. Ce ralentissement, prévu et recherché par les créateurs de BD, peut être suivi d'une accélération grâce à une séquence de petites cases évoquant une scène agitée; cette alternance détermine un rythme de lecture à géométrie variable, qui produit des modalités de réalisation et lecture tensive (BARBIERI, 1991).

STRATE DE LA SENSORIALITÉ EFFECTIVE OU SUGGÉRÉE: LES CINQ SENS DANS LES BANDES DESSINÉES. On regarde une BD avec les yeux. Mais l'audition est également en jeu: avec les textes dans les cartouches de légende et dans les bulles, et avec les onomatopées graphiques. Selon leur style, leur taille et leur couleur, ils suggèrent différentes sonorités, exhortant ainsi le lecteur à une formulation intérieure de sons particuliers.

Le goût et l'olfaction, a priori hors de propos concernant l'expérience de lecture d'une œuvre de $\mathrm{BD}$, peuvent être influencés par un processus de réminiscence sensorielle, produit non seulement par le déroulement de l'histoire (ce qui serait, en principe, pas si éloigné de ce qui se passe dans la lecture d'un roman) mais aussi par des procédés purement graphiques comme la stylisation visuelle de l'air, des parfums et des températures. Le toucher est impliqué plus souvent qu'on ne le pense, en particulier dans les BD érotiques, à travers des processus de projection. Par exemple, les mains d'un personnage, dessinées de manière à ce qu'il touche un partenaire sexuel, "deviennent " en toute logique les mains du 
lecteur: ainsi, des fantasmes sensoriels peuvent être impliqués, également grâce à des détails tels que le rendu des formes des corps (PELLITTERI, 2007, 2013).

Une activation plus forte ou plus faible des sens/sensations du lecteur repose souvent sur le genre narratif. Notons que, dans tous les cas, la vue et l'ouĩe sont toujours les sens les plus impliqués, en raison de la structure sémiotique intime des BD en tant que langage basé sur l'image.

\section{IMAGINATION AUDITIVE DANS LES BANDES DESSINÉES: PROPOS PRÉLIMINAIRES}

\section{De la vision du Son}

Il existe, dans la BD, une différence fondamentale entre les signes graphiques destinés à raconter et à décrire des éléments visuels et ceux concernant les phénomènes sonores. Les premiers, à bien des égards, sont issus des déformations optiques introduites par les dessinateurs de BD depuis le début du $\mathrm{XX}^{\mathrm{e}}$ siècle comme conséquence de l'invention de la photographie et, plus tard, du cinéma. Pensons par exemple à une photo présentant une image floue ou d'autres effets visuels et cinétiques dus à la durée d'exposition du film: au fil du temps, les dessinateurs ont reproduit, imité ou se sont inspirés de tels effets, en créant progressivement des versions originales de ces concepts visuels et en les adaptant à la BD (IMAGE [\&] NARRATIVE, 2015).

Sur le plan sensoriel, un paradoxe peut être signalé dans le contraste entre la caractéristique visuelle d'une onomatopée graphique et la sonorité de son sens, le bruit interne que les lecteurs construisent dans leur esprit, instruits et guidés par le rendu graphique de ce bruit. Quand on regarde les lettres simples qui, assemblées, forment un mot ou un mot onomatopéique, en le déchiffrant et en lui donnant un sens, nous " lisons un son ". Ainsi, le mot | maison |, c'està-dire la séquence des lettres et les phonèmes associés $|\mathrm{m}|,|\mathrm{a}|,|\mathrm{i}|,|\mathrm{s}|$, $|\mathrm{o}|,|\mathrm{n}|$, nous suggère inévitablement le concept correspondant et une ou plusieurs images mentales d'une maison (ECO, 1984a). Mais dans un album de BD ou dans un comic book, il y a en plus. C'est un personnage de l'histoire, ou le narrateur, qui prononce le mot | maison |, donc le lecteur est invité à penser aussi à une voix, à un accent et à une intonation: à des variables intensives, suggérées par des variables extensives.

Dans les $\mathrm{BD}$, le lettering ou lettrage est probablement la variable extensive la plus influente dans la définition d'une voix ou d'une " atmosphère sonore ", mais curieusement - elle est aussi la moins exploitée. Habituellement, à la fois dans les cartouches de légende et dans les bulles, la police (font) utilisée est en majuscule (j'en reparle plus en bas). Le texte écrit est ainsi facilement lisible, mais impersonnel et ne permet pas de différencier les voix supposées des personnages. En d'autres termes, le lettrage dans les BD ne fait pas grand usage de styles calligraphiques ou de polices typographiques variées. La raison généralement exprimée par les professionnels des BD est qu'un lettrage uniforme donne une unité et une propreté à la composition globale. Néanmoins, les lecteurs peuvent parfois apprécier des BD dans lesquelles la conversation de personnages particuliers est connotée avec un type de police spécifique, telle que la caroline médiévale, un alphabet gothique etc., afin de recréer des atmosphères narratives, tout au long desquelles les lecteurs peuvent constituer, dans leur esprit, 
des voix et des timbres particuliers, ou même des effets auditifs (échos, par exemple).

Pour faire passer l'idée qu'un personnage raconte son histoire à la première personne, les lettreurs utilisent parfois une police calligraphique ou un style de police manuscrite qui suggère que ce texte doit être lu mentalement avec la voix imaginée du personnage qui a composé ces lignes. Dans d'autres cas, la police utilisée est une simulation de lettres dactylographiées, pour donner l'idée de textes provenant de documents officiels ou d'articles de presse; dans le cas d'un robot ou d'un ordinateur, les lettreurs utilisent plutôt des polices destinées à suggérer l'usage d'une technologie du futur etc.

Mais le lettrage dans la BD ne doit pas nécessairement " parler ": il n'est pas obligatoire que les polices suggèrent explicitement une correspondance auditive. C'est précisément là que l'imagination auditive intervient: les lecteurs s'engagent, en appréciant un récit, dans la formulation des voix internes imaginaires de tous les personnages de l'histoire. Ce qui est propre à la BD, toutefois, c'est que l'imagination auditive ne provient pas du texte alphabétique conçu comme une suite conventionnelle de symboles qui ont une signification linguistique (comme dans un roman), elle provient ou peut provenir des symbolismes graphiques et de la narration visuelle.

\section{De l'écoute du Silence}

Lorsque nous lisons une $\mathrm{BD}$, nous sommes censés plonger en partie dans sa dimension diégétique. C'est donc aux éléments graphiques d'influencer les lecteurs pour qu'ils puissent comprendre non seulement une onomatopée visuelle, comme | ka-boom | qui indique "explosion ", mais aussi que c'est un type spécifique d'explosion: on peut en déduire qu'elle est divisée en deux sons, le premier étant plus court et puissant, le second ayant une tonalité différente et plus longue. C'est le " travail " du lecteur de spécifier dans son esprit les bruits qu'il veut entendre, en les extrayant de son catalogue personnel de bruits issus de ses expériences passées.

Mais un album ou une BD peuvent être ce qu'ils sont sans le moindre besoin de textes. Un exemple célèbre est celui de la page 23 de The Dark Knight Returns de Frank Miller (DC Comics, 1986), dans laquelle nous autres lecteurs voyons le meurtre des parents du jeune Bruce Wayne par un voleur dans une ruelle de Gotham City et aucun mot ou son n'est affiché2 ${ }^{2}$.

Les BD sans mots ont été introduites au début du $\mathrm{XX}^{\mathrm{e}}$ siècle. Je me limiterai ici à n'en donner qu'un seul exemple, une BD d'une page de Raymond de la Nézière (1904) (SAUSVERD, 2009a). On y voit deux écoliers s'amusant avec un phonographe: les enfants enregistrent dans l'appareil une leçon d'école qui sera récitée plus tard devant le professeur, émettant les voix enregistrées dans le but de le duper. Il n'y a pas d'indicateur de ces voix et de ces sons, mais nous pouvons imaginer qu'ils sont présents à partir des actions des personnages.

2 DC Comics n'a pas répondu à ma réquête de permission de réproduire l'image. Mais les lecteurs peuvent examiner cette célèbre séquence au lien: https://bigother.com/2010/03/15/reading-frank-millers-batman-the-dark-knight-returns-part-3/page-23-2. 
DOSSIÊ

Figure 1 - Raymond de la Nézière, La Leçon dans le Phonographe, 1904

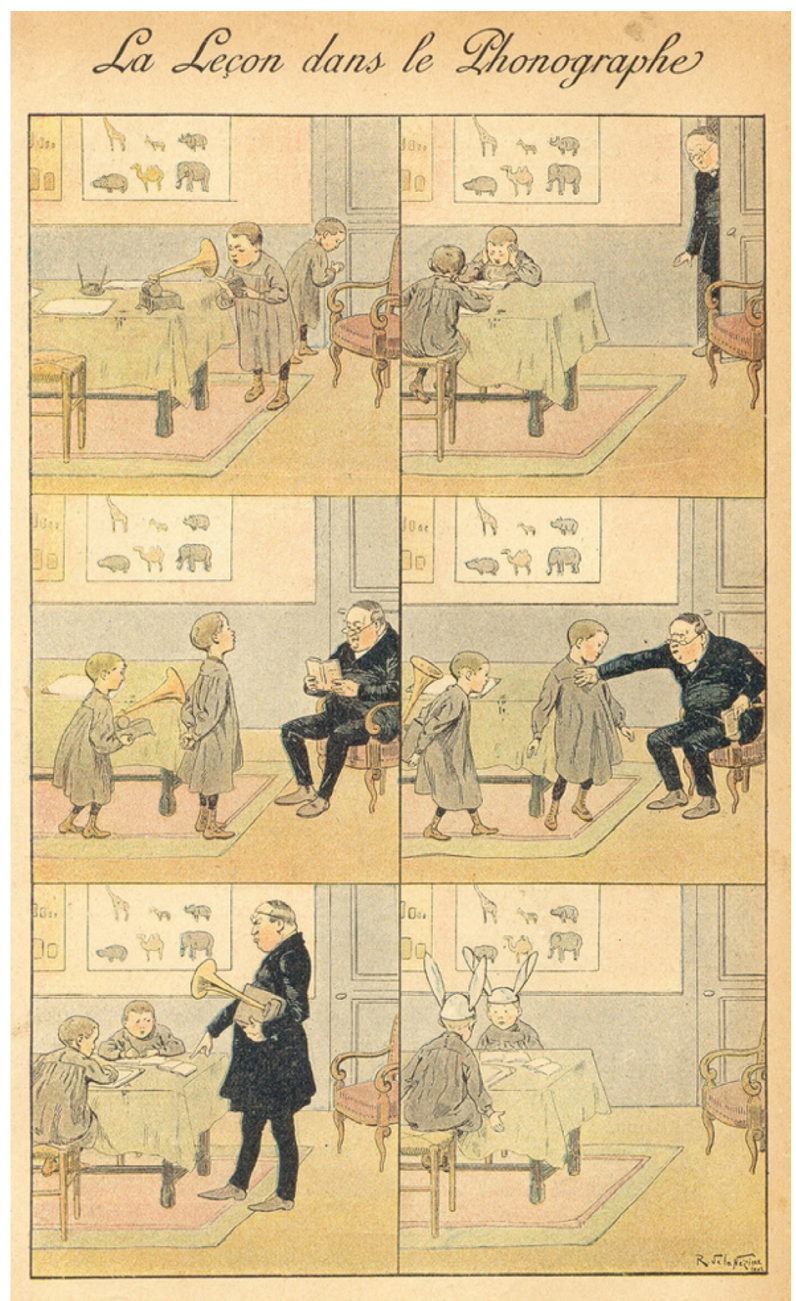

Un art narratif graphique qui n'utilise pas les mots est le roman sans parole ou le roman gravé sur bois, inauguré par Frans Masereel (1919) avec ses 25 images de la passion d'un homme. Celui-ci et les nombreux autres qui ont suivi, de Masereel et d'autres artistes tels que Lynd Ward ou Otto Nückel, ont été des tentatives de construire des contes longs et dramatiques dont le récit se déroulait sans parole. Plutôt que des séquences, les illustrations de ces contes sont organisées en moments, montrant un récit cohérent. Des artistes à l'œuvre de nos jours tels que Eric Drooker, Thomas Ott, Peter Kuper et Shaun Tan ont marché dans les pas de ces premiers auteurs de romans sans parole ${ }^{3}$.

3 Parmi les œuvres des auteurs mentionnés: Drooker (1992), Ott (1997), Kuper (2003) et Tan (2007). Sur les romans sans mot, cf. Beronä (2008) et Tosti (2016, p. 504-521). 
Figure 2 - Une planche de Frans Masereel, 25 images de la passion d'un homme, 1919

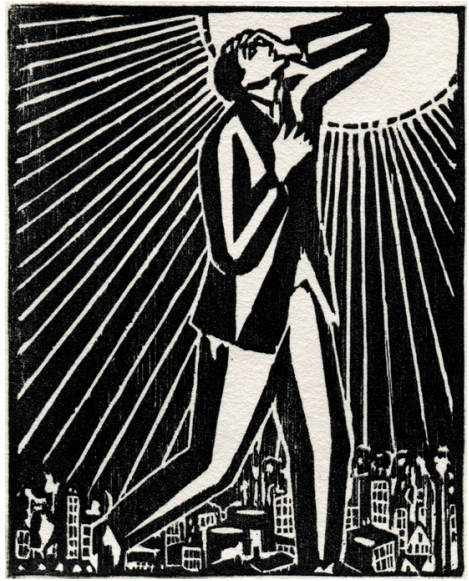

\section{SuR LE LETTRAGE DES BANDES DESSINÉES}

\section{Remarques générales}

Le lettrage trouve ses origines dans le domaine de la calligraphie et est devenu plus tard un métier graphique dont les applications couvrent une grande variété de domaines (BRINGHURST, 1992; TINSLEY, 1999, p. 39-76; STARKINGS; ROSHELL, 2003; CHIARELLO; KLEIN, 2004, p. 82-141; PELLITTERI, 2012, p. 263-265). Les lettreurs véhiculent des caractéristiques spécifiques du sens suggéré du texte en utilisant une diversité de moyens graphiques.

Comme nous l'avons vu aussi, il a toujours été plus facile pour les dessinateurs ou les lettreurs d'écrire en majuscules. Quelques exceptions existent, par exemple les albums Tintin d'Hergé (1929-1975). De manière générale, les caractères en italique et en minuscules sont utilisés dans les albums pour transmettre des nuances spécifiques: par exemple, une manière élégante et calme de parler; utilisés systématiquement, ils donnent à l'histoire un charme plus littéraire, comme dans Blake et Mortimer (1946-1990) d'Edgar P. Jacobs. L’imagination auditive est impliquée ici parce qu'un lettrage élégant utilisé, par exemple, pour un lord britannique, suggère que l'élocution et l'attitude du personnage peuvent être altières: le lecteur sera invité à lui conférer un certain accent et des manières raffinées.

Le lettrage peut également consister, encore aujourd'hui, dans la création de nouvelles formes et codes sémiotiques pour les bulles de textes: il est toujours possible d'innover dans leurs fonctions. Les principaux contributeurs à l'innovation dans ce domaine sont le regretté maître de la BD Will Eisner ainsi que Dave Sim, Todd Klein (EISNER, 1985; THOMAS, 2000) et quelques grands artistes japonais tels que le défunt Osamu Tezuka ou encore Sanpei Shirato ${ }^{4}$.

4 Des catalogues et analyses des bruits et des sons dans les bd et les manga, ainsi que leurs visualisations, se trouvent dans Khordoc (2001), Pollmann (2001), Posocco (2005), Covey (2006) et Petersen (2009). 


\section{Le lettrage, des premières bandes dessinées aux bandes dessinées contemporaines}

La présence de textes dans des compositions figuratives remonte au $\mathrm{XV}^{\mathrm{e}}$ siècle, sous forme de phylactère. Les étapes de développement dans l'utilisation de phylactères et de phrases écrites montre les canaux historiques qui ont mené aux modalités actuelles d'insertion de textes verbaux dans des contextes iconiques; à partir de la fin du $\mathrm{XIX}^{\mathrm{e}}$ siècle, des versions révisées des phylactères, devenues des bulles de textes, devinrent l'outil de transmission des messages verbaux ou des pensées dans les BD. Avec le développement des caricatures politiques en Grande-Bretagne, l'usage des bulles et du lettrage dans les dessins humoristiques et satiriques est devenu familier du public et a constitué une base sur laquelle les artistes ultérieurs, dans d'autres pays européens également, ont commencé à mélanger des illustrations avec divers types de textes alphabétiques (KUNZLE, 1973; CASTELLI, 2006). Des exemples notables viennent non seulement de la Grande-Bretagne, mais aussi des États-Unis et de Suisse (COUPERIE, 1968, p. 7-33). En d'autres termes, le texte verbal a commencé très tôt à être couplé aux dessins; dans un premier temps, en tant que co-présence dans des zones contiguës de l'illustration; plus tard, dans une association plus étroite.

Aux débuts du médium BD dans son sens moderne et au cours des décennies jusqu'aux années 1940 (BECKER, 1959, p. 1-53), le lettrage était effectué par les dessinateurs eux-mêmes ou, dans le cas des auteurs les plus productifs et célèbres, par un assistant. C'est à partir des années 1940 qu'une division du travail a été développée au sein des studios et agences de BD (MCCLOUD, 2006, p. 128-57, 184-211).

\section{Bulle (ou ballon) de textes}

Au cours des dernières années, les chercheurs internationaux ont convergé vers l'idée que la définition des BD repose sur d'autres éléments structurels que la bulle (FORCEVILLE et al., 2010). Il est utile de fournir une série d'exemples de la façon dont elles véhiculent des indices auditifs.

Clarifions d'abord une distinction entre bulle de textes et bulle de paroles. Les bulles de paroles renvoient aux dialogues parlés provenant de personnages qui sont "en scène "; elles sont un sous-groupe des bulles de textes. "Bulle de textes ", en règle générale, désigne toute bulle contenant des mots - parmi lesquels figurent, par exemple, des pensées ou des sources artificielles de sons. Les lecteurs déduisent facilement, à la forme de leur contour, quels sont les différents types de bulles.

Aujourd'hui, la bulle et le texte qu'elle contient sont considérés par beaucoup comme quelque chose de "naturel " dans la BD. Cependant, cette notion doit être profondément repensée. Le prédécesseur des actuels bulles de texte et cartouches de légende est, comme vu précédemment, le phylactère. ${ }^{5}$ Une étude de 1'historien belge Thierry Smolderen (2006) analyse les étapes qui ont conduit les bulles à être utilisées comme un outil directement associé aux personnages qui parlent. Il s'avère que ces dispositifs étaient étrangers à la BD en tant que lan-

5 En 1868, Baudelaire décrivit comme un vieux procédé I'utilisation de ce qu'il appelait, en référence au caricaturiste français Grandville, les banderoles parlantes (BAUDELAIRE, 1868, p. 411). 
gage: ils ne communiquaient au lecteur aucun son possible ou aucune idée de son, mais seulement des textes abstraits, non sonores. Même les premiers maîtres de la BD et des illustrateurs de renom du XIX siècle, comme Töpffer, Cham, Gustave Doré et Casimiro Teja (GADDUCCI, 2006) ont rarement fait usage de ce procédé graphique. Ainsi, en un sens, la plupart des premières BD ne possédaient pas ce mélange de texte verbal et de texte iconique, qui est une caractéristique fréquente de la BD contemporaine.

Dans les premières BD européennes, par exemple, l'utilisation des bulles était souvent maladroite, mais toujours intéressante et très efficace, en suggérant la nature auditive du texte qu'elles contiennent. Dans une histoire d'une page de l'artiste français Benjamin Rabier (1907), les bulles provenant d'un phonographe sont présentées comme de " nuages sonores " moelleux plutôt que comme des récipients symboliques de textes verbaux, et l'effet général pour le lecteur est assez intrigant (SAUSVERD, 2009b).

Figure 3 - Benjamin Rabier, Le Phono-Piège, 1907

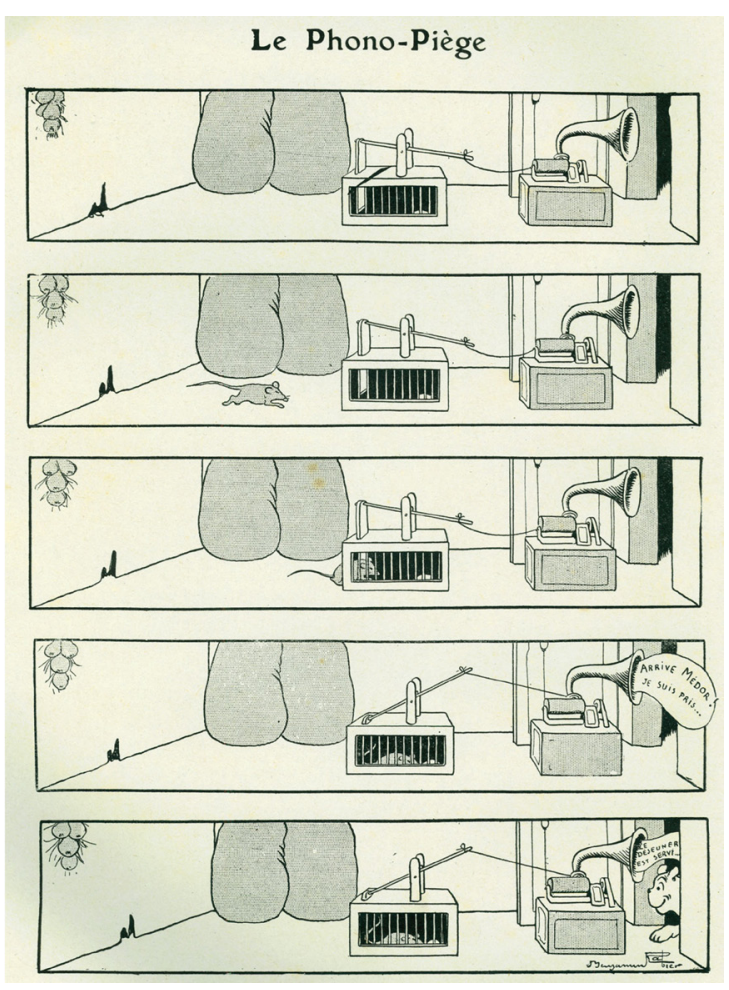

Dans une autre proto-BD française, les bulles sont absentes et le dialogue entre deux utilisateurs précoces du téléphone est rendu à travers des textes présentés presque comme des ondes sonores. Cette page d'Albert Guillaume de 1894 s'articule en neuf cases dont trois, la colonne centrale, ne montrent que les mots prononcés par les deux personnages principaux au cours de leur conversation: de tels textes fluctuent à l'intérieur des cases et sont reliés par contiguïté avec les appareils respectifs, lesquels sont pratiquement accrochés aux contours de division des cases. 
DOSSIÊ

Figure 4 - Albert Guillaume, Les Perfidies du Téléphone, 1894

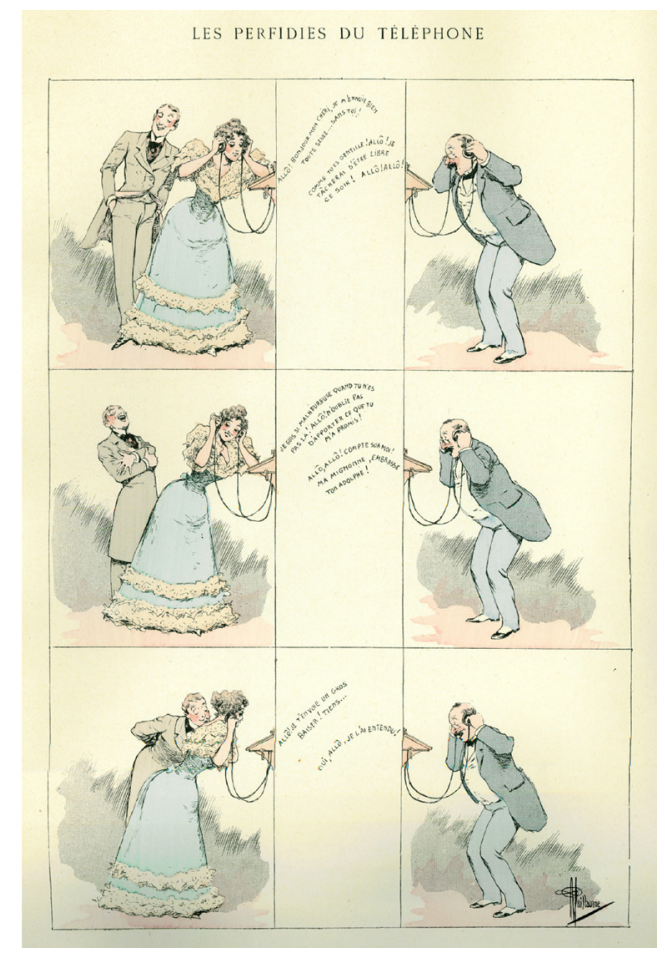

Peu à peu, cependant, les bulles ont, d'une façon générale, commencé à jouer le rôle de voix et de sons diégétiques. Le mot est devenu un élément clé pour créer chez le lecteur le sentiment que l'histoire se déroule au fil du temps. Le temps de lecture lui-même a donné aux événements dans les cases une durée de narration réaliste. Pour que cela se produise, les lecteurs ne devaient pas reconstituer dans leur esprit une abstraction muette de ces textes, mais devaient plutôt inventer des voix et des expressions pour donner de la crédibilité à ce qu'ils voyaient et aux personnages.

L'apport créatif des bulles de textes "fournit des informations sur ou améliore 1. la manière de parler; 2. le sujet du discours; et/ou 3. l'identité du locuteur. Il existe un continuum allant des utilisations complètement conventionnelles aux utilisations hautement créatives des variables que sont les bulles " (FORCEVILLE, 2013, p. 268). Les bulles de BD, tout comme les cartouches de légende, ne sont guère exploités dans une perspective auditive: les textes qu'ils contiennent sont presque toujours " neutres ", à l'exception de certains mots saisis en gras. La ponctuation est, par contre, largement utilisée: notamment dans les BD humoristiques, les phrases se terminent souvent par un point d'exclamation, pour souligner un ton amusant et/ou énergique. Même sans tenir compte de la possibilité que les textes contenus dans les bulles soient rendus par des polices ou des tailles différentes. Une façon très commune de suggérer différents styles de conversation, des énergies, des volumes et des attitudes divers, tient dans les formes des bulles: les lecteurs considèrent généralement que les bulles hérissées de pointes aiguës sont des " bulles de cris " et que les bulles 
à contour en pointillés signifient que le personnage associé murmure ou bavarde, selon la situation narrative.

\section{ONOMATOPÉES GRAPHIGUES: L'EXPRESSION VISUELLE DES BRUITS DANS LES BANDES DESSINÉES}

Les onomatopées ont été prises en compte, cataloguées et étudiées dans de nombreux travaux universitaires européens, en particulier depuis les années 1970, au cours d'un boom de l'analyse structuraliste (NENCIONI, 1971), inspiré par l'essai précurseur d'Umberto Eco "Lettura di Steve Canyon " (ECO, 1964). Encore aujourd'hui, les linguistes européens qui apprécient aussi la BD comme sujet d'étude réalisent des enquêtes approfondies sur les onomatopées verbales et visuelles dans les BD (p. ex. PIETRINI, 2009; FRANCESCHI, 2009; GADDUCCI; TAVOSANIS, 2009).

\section{Le rôle des onomatopées pour l'imagination auditive dans les bandes dessinées}

Les onomatopées des BD peuvent être de trois types: 1. les vocalisations provenant des personnages et généralement (mais pas toujours) placées à l'intérieur des bulles de textes en tant que textes verbaux; cependant, dans ce cas, nous ne parlons pas d'onomatopées visuelles mais d'onomatopées verbales; 2. effets sonores/de bruit, rendus visuellement sous la forme d'un "logo " en lettres, ou d'une "étiquette " apparaissant comme des éléments distincts dans la case ou entre les cases; et 3. dans certains cas, des onomatopées visuelles qui sont des cases en forme de mot, à l'intérieur desquelles l'action se déroule; par exemple une explosion dessinée et décrite à l'intérieur d'une case Boom en forme de logo/d'étiquette, ou Blam etc.

À cette fin, la délimitation des différentes catégories d'onomatopées dans les $\mathrm{BD}$ " signale une différenciation interne au sein de l'industrie de la BD quant à la nature du son représenté graphiquement et parle peut-être aux limites du rendu des phénomènes auditifs dans des médias non auditifs " (GUYNES, 2014, p. 61). Cette notion s'étend aux onomatopées conçues comme des imitations ou des suggestions de bruits naturels, et ici le discours devient encore plus pertinent par rapport à la notion de son et d'imagination. En soi, les onomatopées sont des approximations symboliques des bruits et des sons naturels: bien qu'il n'y ait pas de correspondance directe entre les mots ou les combinaisons de lettres comme | boom |, | pow |, | ka-blam | ou d'autres et le bruit réel d'une explosion, les lecteurs sont à même de reconstituer dans leurs propres pensées des versions personnelles de ces sons, en faisant référence à des expériences passées et à d'autres médias où ces sons sont effectivement entendus. Les lecteurs ont une liberté et une variété infinies de choix à l'intérieur du champ sémantique général d'une onomatopée donnée. Alors que le mot écrit | Boom | est le même pour tout le monde, chaque lecteur est en mesure de, et encouragé à, recomposer dans son esprit (ou, pour des lecteurs plus jeunes et proactifs, peut-être même à reproduire avec leur propre bouche), le bruit spécifique qui est à son avis le bon pour cette occasion particulière. Il est donc plus facile de s'accorder sur le fait que les onomatopées dans les BD "créent un intérieur animé pour que l'histoire y soit vivante ", un " espace pour que le sens s'y accroisse " (PETERSEN, 2009, p. 165). 


\section{Grands bruits, petits bruits, effets sonores génériques}

Dans I linguaggi del fumetto, Daniele Barbieri (1991, p. 173-174) appelle les onomatopées visuelles des BD des "grands bruits". Les grands bruits sont des phénomènes tels que les explosions déjà mentionnées ou d'autres sons tels que, par exemple, le crissement soudain et prolongé de pneus sur l'asphalte d'une route pendant une poursuite. Dans ces cas, le mot/son onomatopéique, en anglais, sera un screech classique ou de nouveaux sons inventés par les auteurs pour l'occasion, comme skreeeeeee dans Sin City de Frank Miller (1991) ${ }^{6}$. Cette relation variable entre le son et les onomatopées visuelles produisent des combinaisons infinies, comme vu précédemment. Dans la BD humoristique (par exemple, dans la tradition remarquable des BD de Disney faites en Italie), le son d'une pile d'assiettes que les mains de Donald Duck laissent se briser bruyamment au sol pourrait ressembler à sbadabumpumcrash!: dans ce cas, le lecteur est informé que le bruit est prolongé et formé de plusieurs étapes successives; les lecteurs ont donc, pour consigne de former dans leur esprit, un son probablement drôle, composite et assourdissant ${ }^{7}$.

Outre les grands bruits, les textes de cartouches de légende et les bulles de texte, de nombreuses BD produisent également des effets destinés à représenter des sons particuliers tels que des tonalités musicales ou mélodies, bourdonnements, bredouillements, par exemple. Je vais mettre l'accent sur la musique plus bas, dans une section dédiée. Les bruits de fond et les effets sonores sont communiqués au lecteur de deux façons: la première est un moyen direct et consiste en de petites onomatopées graphiques, uniques ou répétées; elles sont généralement placées au milieu ou au bas d'une case. La seconde, indirecte, est un dispositif purement diégétique: aucun élément graphique n'est affiché pour indiquer le bruit, la case est muette, ou bien présente des symboles graphiques ou des motifs qui ne sont pas sous forme de mots alphabétiques. Un vent qui souffle, un tic-tac d'horloge, un grincement de porte sont autant d'éléments d'une certaine importance pour une scène, et une telle scène ne doit pas nécessairement comporter des onomatopées graphiques: des bruits peuvent également être suggérés, comme nous l'avons vu précédemment, en termes diégétiques et à travers des symboles graphiques qui peuvent grosso modo se rattacher au domaine de l'alphabet.

Les BD japonaises présentent souvent des déclencheurs graphiques aux suggestions synesthésiques. Dans Video Girl Ai de Mazakazu Katsura, l'imagination intra-diégétique du protagoniste, un adolescent, se présente souvent sous la forme de symboles typographiques ou calligraphiques et de métaphores visuelles: un sentiment de doute est exprimé par un arrière-plan rempli de points d'interrogation; une bulle de pensée, avec des considérations sur la possibilité de rencontrer à la piscine une jolie fille dont il est tombé amoureux, possède un contour complexe, agrémenté de nombreuses petites bulles, symbolisant l'enthousiasme suscité par l'éventuel événement; et une case montrant la visualisation, dans l'esprit du garçon, de la fille qui porte son maillot de bain moulant, est agrémentée d'un arrière-plan montrant un soleil stylisé avec des rayons, ce

6 Les lecteurs peuvent vérifier et examiner cette onomatopée au lien suivant: Ebaumsworld.com/pictures/sin-city-the-hard-goodbye-episode-7/81114520 (page 2 sur 8).

7 Dans de tels cas, la multimédialité, les possibilités multi-sensorielles et l'imagination auditive des bd se mêlent à une certaine intertextualité, en ce que les lecteurs possèdent peut-être des souvenirs personnels, en tant que spectateurs de cinéma ou de télévision, de courts métrages d'animation classiques de Disney dans lesquels Donald Duck a des ennuis et où l'issue de sa négligence est un dégât majeur pour les objets: cela se produit, par exemple, dans Cured Duck (7', 1945, de Jack King). 
qui représente l'excitation réelle du garçon. Si le lecteur devient complice de façon proactive avec le but de l'histoire, il sera amené à imaginer une pluralité de petits effets sonores de courte durée qui serviront de commentaire auditif au développement du récit: halètements répétés de surprise, bruits légers de petites bulles d'eau ou de savon qui explosent, note de trompette affirmée ou " tadaa! " générique correspondant à la case contenant la fille et l'arrière-plan ensoleillé. Le choix, bien sûr, dépend toujours du lecteur.

Figure 5 - Masakazu Katsura, Den'ei Shōjo (titre international: Video Girl Ai), page 22 du premier volume de l'édition américaine la plus récente. Avec l'aimable autorisation de: VIZ Media. VIDEO GIRL AI (C) 1989 by Masakazu Katsura/ SHUEISHA Inc.
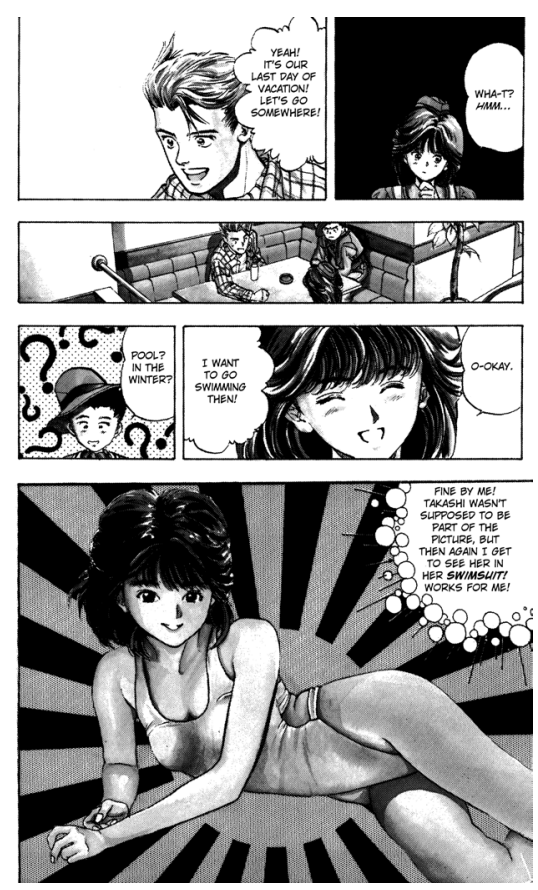

Il existe toutefois des moyens plus subtils de suggérer des bruits de fond ou des sonorités d'atmosphère. Je traiterai de ces alternatives dans la section finale.

\section{Les onomatopées dans les bandes dessinées " de frontière »}

Je voudrais souligner deux manières particulières dont les onomatopées comiques ont été utilisées dans des œuvres d'art liées à la musique et qui peuvent, jusqu'à un certain point, être qualifiées de " quasi-BD ". Manifestement, les langages et conventions graphiques des BD ont été utilisés dans de nombreux médias: cinéma, télévision, design et publicité, animation, affichage public et, récemment, internet et la messagerie instantanée numérique. Mais ici, je ne parlerai que de la façon dont les BD ont été utilisées pour exprimer la musique et le bruit dans l'art contemporain. Par conséquent, cette digression peut être lue comme un petit corollaire à la section suivante sur la musique. 
Dans certains mouvements artistiques de la seconde moitié du $\mathrm{XX}^{\mathrm{e}}$ siècle, inspirés de mouvements historiques avant-gardistes tels que le Futurisme et le Surréalisme, certains artistes ont tenté de développer de nouvelles manières d'écrire la musique, pour transformer le temps musical en figuration. Ces expérimentations se sont croisées avec les BD (TORELLI LANDINI, 2012). Tel est le cas, par exemple, de Telephone Blues Opera K. 731 (1974), de Luciano Ori: une partition musicale dans laquelle des bulles de textes et des onomatopées graphiques apparaissent littéralement issues du groupe de musiciens, sous forme de commentaires bruyants sur la partition principale ${ }^{8}$.

Plus convaincant, cependant, se trouve être le cas de Stripsody per voce sola (BERBERIAN, 1967), une composition de 1966 de la chanteuse mezzo-soprano Cathy Berberian: une performance basée sur la reproduction vocale des onomatopées (LINDEKENS, 1976). Cette performance artistique a inspiré deux ouvrages visuels: l'un d'Eugenio Carmi, la même année; l'autre de Roberto Zamarin, en $1967^{9}$. Les travaux graphiques de Carmi et Zamarin ont pour but de restituer, à travers des compositions conceptuelles géométriques, la puissance auditive des onomatopées comiques (GARBUGLIA, 2011) ${ }^{10}$.

Figure 6 - Eugenio Carmi, rendus graphiques de Stripsody per voce sola ('Stripsodie pour soliste') de Cathy Berberian, 1966. Avec l'aimable autorisation de l'Eugenio Carmi Archive

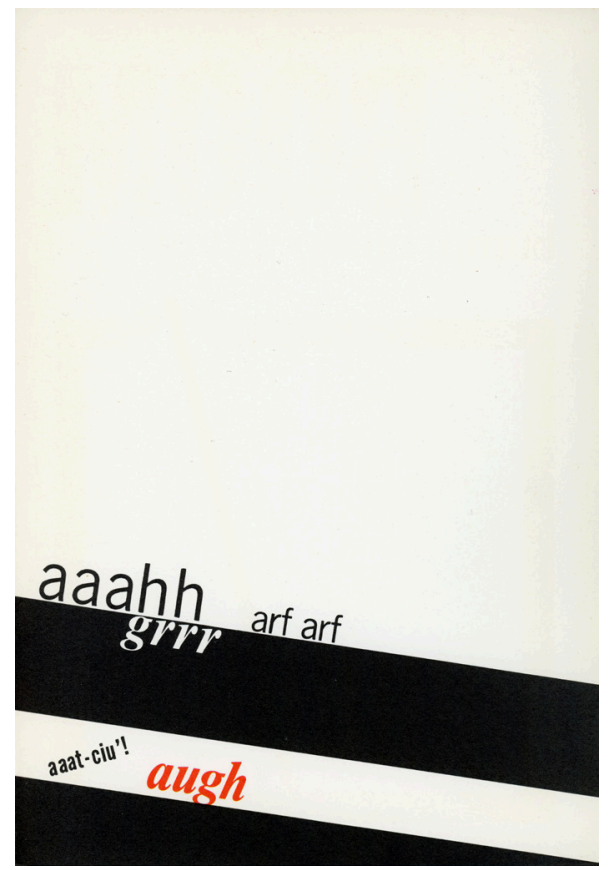

8 Les lecteurs peuvent examiner les ouvrages d'Eugenio Carmi, y compris le travail cité ici, à ce lien: http://continuo-docs.tumblr. com/post/17886579731/imaginary-music-scores-by-visual-poet-and.

9 Les lecteurs peuvent facilement consulter par eux-mêmes plusieurs pages du travail graphique de Zamarin ici: https://musicomix. wordpress.com/stripsody.

10 Un enregistrement de l'une des performances de Stripsody se trouve, par exemple, ici: https://youtu.be/rmOwX1xTAak. Pour un examen plus approfondi de Stripsody et des travaux de Carmi et Zamarin, voir: https://musicomix.wordpress.com/stripsody. 
Figure 7 - Eugenio Carmi, rendus graphiques de Stripsody per voce sola ('Stripsodie pour soliste') de Cathy Berberian, 1966. Avec l'aimable autorisation de l'Eugenio Carmi Archive
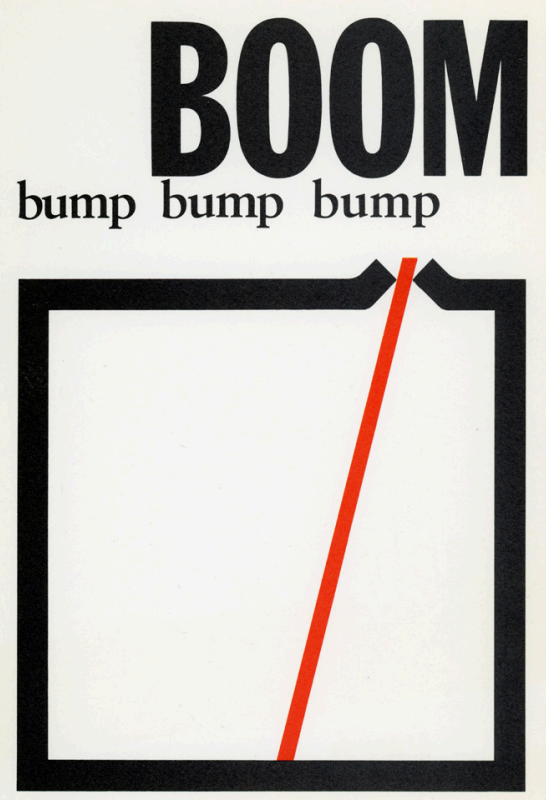

Voyons maintenant comment les vraies BD traitent de cet aspect du son et de l'imagination.

\section{LA MUSIgUe DANS LES BANDES DESSINÉES}

Selon Richard Wagner et son idée d'un Gesamtkunstwerk, une " œuvre d'art total " pourrait unifier toutes les formes d'expression en une seule (dans le cas de Wagner, le théâtre; cf. WAGNER, 1849). Les BD, comme j'ai essayé de le montrer, peuvent être conçues comme un médium complet dans lequel plusieurs couches de communication et de sensorialité peuvent converger dans l'esprit d'un lecteur créatif et coopératif. En un sens, nous pouvons donc dire que les BD peuvent également être conçues comme une forme d'expression " totale " capable d'impliquer le lecteur dans des expériences répétées de lecture synesthésique.

La synesthésie, dans le cas de la musique dans les BD, implique l'affichage de symboles musicaux, de paroles de chansons, de partitions et/ou de situations narratives dans lesquelles les personnages sont clairement engagés dans une activité musicale. Le célèbre auteur de BD Chris Ware, ainsi que son collègue Craig Thompson, ont présenté leurs points de vue sur le sujet (IRVING, 2012; SANTIROSI, 2012). Selon les deux artistes, les BD sont comme une partition musicale, les dessins sont les notes et il appartient au lecteur de décider du rythme: les artistes ne fournissent que les indices.

Comme la musique est rythmique et prosodique, il est important de prendre en compte l'apparition de vers et de strophes dans les premières $\mathrm{BD}$, le plus sou- 
DOSSIE

vent en rimes: dans Töppfer, Doré ou dans des magazines historiques comme la revue italienne Corriere dei Piccoli (1908-1995) et la revue espagnole Dominguín (1915-1916).

Figure 8 - Première page du premier numéro italien de Corriere dei Piccoli ('La Gazette des Petits'), 27 Décembre 1908; le supplément hebdomadaire pour enfants du quotidien Corriere della Sera

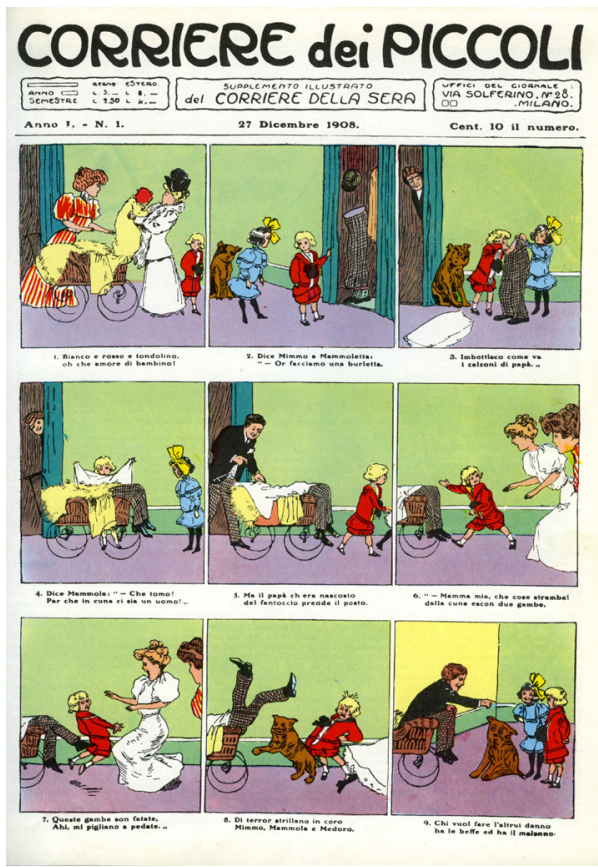

Figure 9 - Première page du premier numéro de l'hebdomadaire espagnol Dominguín, 12 Décembre 1915

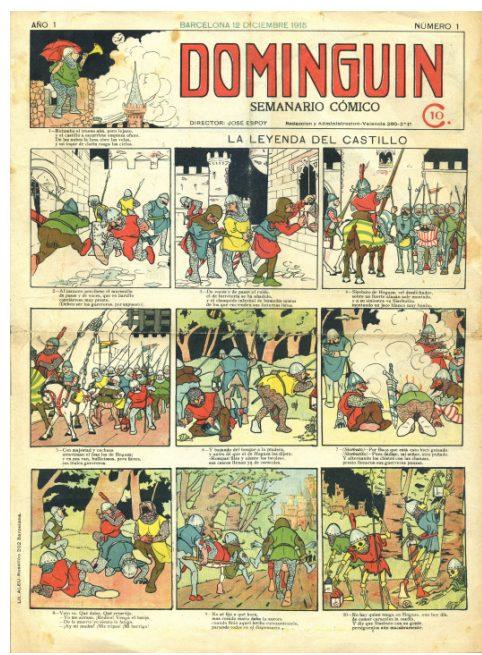


La principale raison des vers rimés comme commentaire narratif d'histoires illustrées, ou de BD publiées à profusion dans les magazines précités, était le contrôle du potentiel culturellement subversif de cette forme de narration visuelle alors nouvelle et perçue comme dangereuse pour les enfants (DETTI, 1984). Une autre raison, plus subtile, était l'intention d'introduire une articulation rythmique, auditive et vocale, afin de subordonner quelque peu le plaisir procuré par les images à la lecture littéraire (TOSTI, 2016, p. 543-58). En fait, la présence de strophes de rimes encourage à lire avec un rythme, donnant à l'expérience de lecture une cadence au flux de notre voix interne déclamant les versets.

Les musiciens et les partitions sont apparus dans les pré-BD, les proto-BD et les premières BD à maintes reprises. Je limiterai les nombreux exemples à seulement quelques-uns: une page du conte illustré de Gustave Doré Des-agréments d'un voyage d'agrément (1851), la planche unique Une Valse de JeanJacques Grandville (1840) et le Der Virtuos de Wilhelm Busch (1865). Dans la composition de Grandville, nous voyons des musiciens anthropomorphisés: les notes sont des petits hommes qui jouent/se battent sur et à travers la partition et donnent un rythme musical particulier à l'ensemble de la situation. Ceci fut source d'inspiration pour la page de Des-agréments de Doré dans laquelle plusieurs références à la musique et aux partitions apparaissent. En ce qui concerne Der Virtuos de Busch, on y voit l'exécution d'un morceau musical par un pianiste: chaque illustration de la composition possède un " titre " spécifique qui constitue les indications relatives à l'exécution du morceau par le pianiste, ce qui crée un contrepoint humoristique entre les vignettes d'orientation écrites et les expressions faciales et mouvements de corps exagérés des deux personnages sur scène (RIES et al., 2007). De plus, la vivacité de la scène et la "matérialité " de la musique sont rendues visuellement par les notes musicales mouvantes, issues de la partition.

Figure 10 - Gustave Doré, Des-agréments d'un voyage d'agréments, 1851

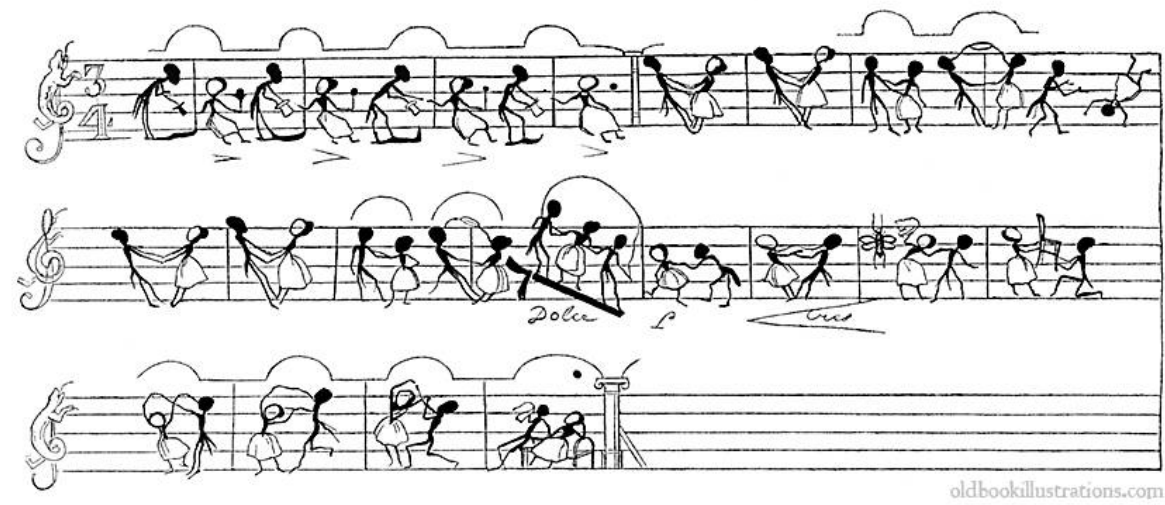


DOSSIÊ

Figure 11 - Jean-Jacques Grandville, Une valse, 1840

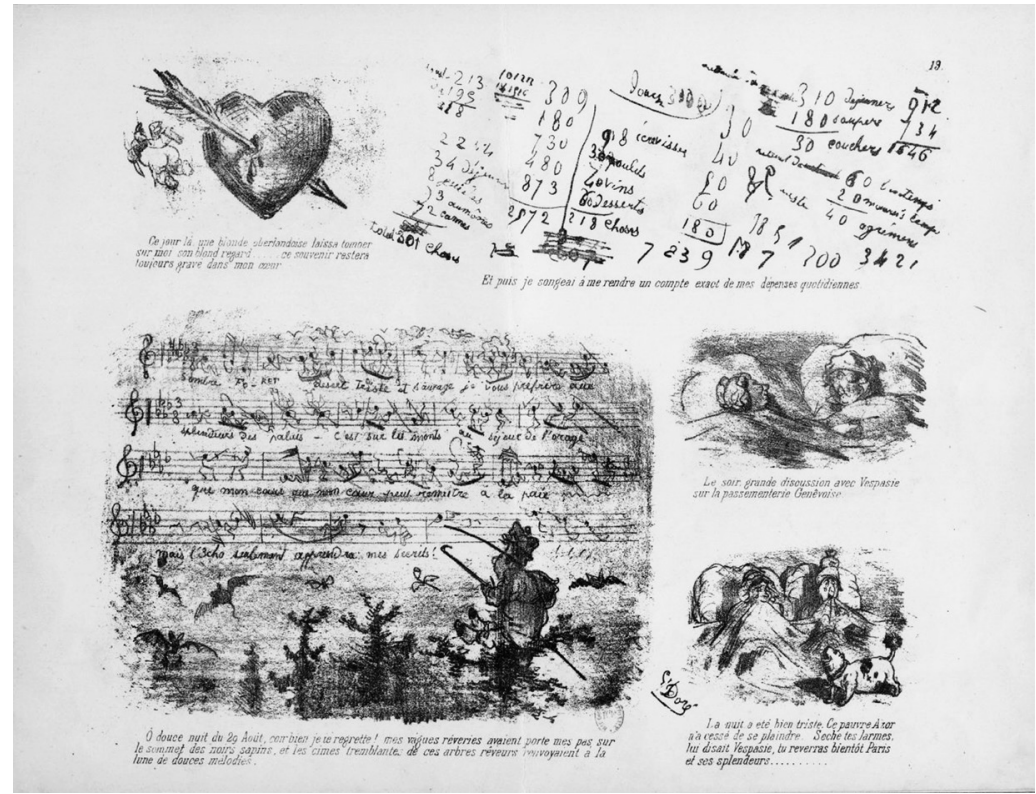

Figure 12 - Wilhelm Busch, Der Virtuos ('Le Virtuose'), 1865

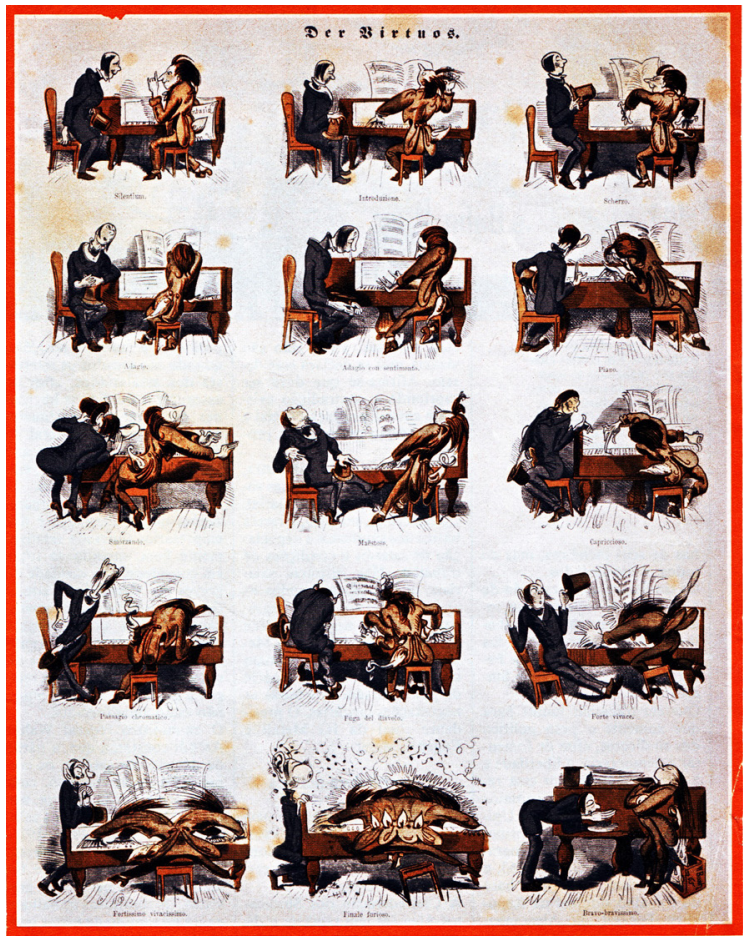


Dans l'histoire de l'art séquentiel, certains ont tenté de traduire en dessins le rythme, la mélodie et le dynamisme d'une partition représentant une danse. Un cas surprenant se trouve être celui de l'artiste française Valentine Gross-Hugo. En mai 1913, la partition de Igor Stravinskij Le Sacre du Printemps, ainsi que les chorégraphies de Vaclav Nižinskij, ont été accueillies avec frénésie au Théâtre des Champs-Élysées à Paris. La même année, Gross-Hugo a dessiné un ensemble de dessins sous forme d'annotations liées aux chorégraphies mises en scène par le célèbre danseur russe. Dans cette série de dessins, nous voyons des esquisses des mouvements du danseur accompagnant des morceaux de la partition, avec des musiciens, des notes etc. On peut voir ici la tentative de synchroniser les morceaux de musique affichés avec la chorégraphie, de manière à guider les lecteurs dans la façon d'imaginer la musique dans leur esprit, tout en visualisant les mouvements du danseur. Le style de dessin utilisé est, en quelque sorte, lui aussi musical: Gross-Hugo a choisi, avec beaucoup d'intuition, de donner vie au danseur par des lignes ondulantes, fines et entrelacées qui rappellent beaucoup le style calligraphique utilisé pour écrire les différents éléments d'une partition (clefs, notes etc.) ${ }^{11}$.

Figure 13 - Valentine Gross Hugo, Le sacre du Printemps, 1913

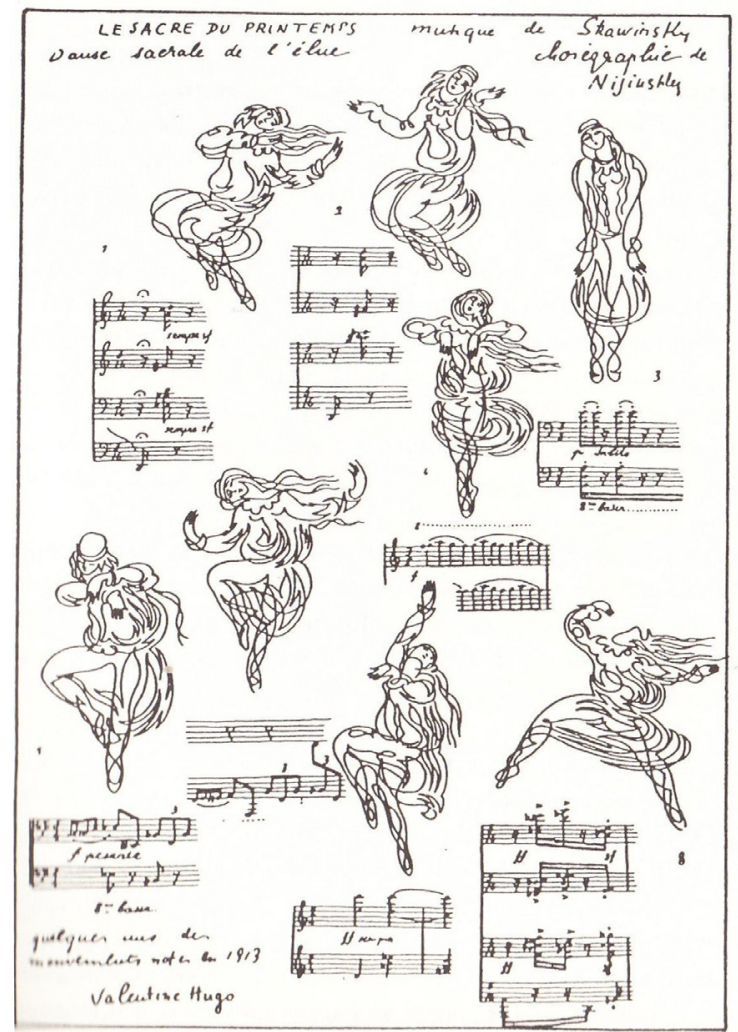

11 On peut trouver des exemples similaires chez Edvard Munch (Tingeltangel, 1895), Pablo Picasso (la couverture de la partition du Ragtime de Stravinskij, 1918-1919) ou la caricature faite par Jean Cocteau de Stravinskij lui-même jouant Ragtime au piano. 
Ceux-ci, cependant, ne sont que des exemples isolés de dessins uniques, réalisés par des artistes peu impliqués dans le monde des BD. À cette fin, j'ai également sélectionné quelques exemples destinés à sensibiliser le lecteur à la complexité et à la richesse des combinaisons possibles entre la musique et la BD.

Dans les BD modernes et contemporaines, les introductions de musique dans les cases et dans l'histoire se produisent principalement à deux niveaux: elles peuvent fonctionner comme bande son extra-diégétique, comme tel est le cas habituellement au cinéma; ou comme bande son intra-diégétique, quand ce sont les personnages qui chantent ou jouent, ou quand, en tant que lecteurs, nous savons par le récit que, dans telle scène, de la musique est présente, car les auteurs nous ont donné des indications ou des informations explicites à cette fin. Dans le numéro 36 (Diritto e rovescio) de la série Ken Parker (1977-2015) de Giancarlo Berardi et Ivo Milazzo (1981); dans Cages (1990-1996) de Dave McKean; ou dans Valentina (1965-1995) de Guido Crepax; ou dans Billie Holiday et d'autres histoires sur le jazz de Carlos Sampayo et José Muñoz (1993); dans tous ces exemples, la musique est protagoniste ou bien possède un rôle important pour un ou plusieurs personnages, donnant à l'ensemble une " ambiance sonore " destinée à plonger le lecteur dans une atmosphère musicale (MASSARUTTO, 2011).

Cependant, profiter pleinement de la présence de la musique dans les BD repose sur un point fondamental, qui est que lorsque des indices ou des partitions d'une chanson ou d'une musique sont donnés au lecteur, le lecteur doit savoir de quoi parle cette musique: connaît-il la mélodie de cette chanson particulière? Est-il capable de lire effectivement la partition si des musiciens avec des notes etc. se trouvent affichés? Sans une prise en compte de ces difficultés potentielles, l'intégration de la musique dans les BD pourrait se révéler presque vaine. En ce sens, les créateurs de BD adoptent deux stratégies principales: 1. Ils ont recours à des compositions qu'ils sont convaincus que leurs lecteurs connaîtront; en d'autres termes, les créateurs de BD veulent partager avec leurs lecteurs une culture et une expérience communes afin de créer plus d'empathie entre eux et le récit; 2 . Les créateurs de $B D$ n'affichent pas de partition spécifique et ne mentionnent pas explicitement les chansons ou la musique que les lecteurs sont censés connaître, mais créent plutôt une atmosphère générale ou donnent aux lecteurs des indications sur le type de musique jouée de manière intra-diégétique dans certaines scènes de l'histoire. Le but est, dans ce cas, de laisser les lecteurs décider de cette musique intra-diégétique (cf. aussi MASSARUTTO, 2011, p. 127-148). Voyons comment ces deux stratégies sont mises en œuvre.

En ce qui concerne la première stratégie, un bon exemple est Delitto al cineclub (1990) de l'artiste italienne Cinzia Leone, dans lequel on voit Rita Hayworth dans Gilda (de Charles Vidor 1946), dans la scène où elle chante ${ }^{12}$ Put the Blame on Mame. Leone utilise des images du film en tant que cases et ajoute des bulles de paroles; elle insère également, dans la partie supérieure de la page, la partition de la chanson. La présence des musiciens est purement décorative, car le lecteur cultivé est censé partager avec l'auteur la connaissance de la mélodie ainsi que celle du film, même s'il n'est pas capable de lire les partitions. Dans les cases inférieures, seules les images du film sont affichées, sans aucun signe supplémentaire: le lecteur connaît déjà l'exercice et peut continuer à imaginer la fin de la chanson et les applaudissements du public sans difficulté.

12 Par souci de précision, la voix n'était pas celle de Hayworth: la chanson était interprétée par Anita Ellis. 
Figure 14 - Cinzia Leone, Delitto al Cineclub ('Meurtre au Cinéclub'), 1990. Avec l'aimable autorisation de Cinzia Leone

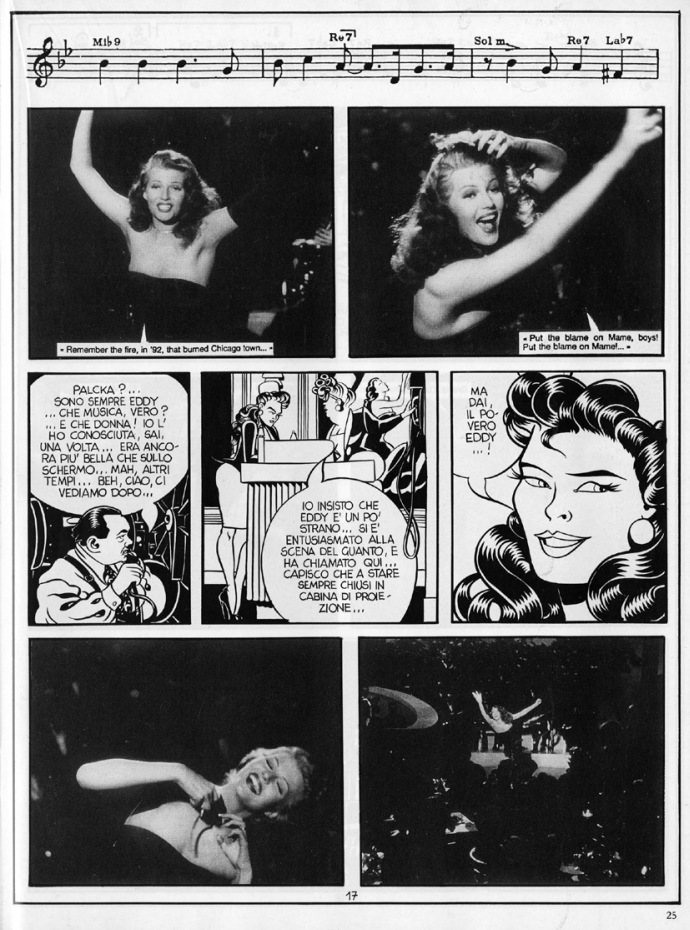

Dans une histoire courte de Milo Manara (1993), John Lennon, l'auteur montre l'ascension au Ciel du Beatle (assassiné en 1980): en quelques scènes l'artiste de Liverpool devient rapidement un chef d'orchestre histrionique qui implique les âmes, les anges et toutes les divinités majeures dans l'exécution chorale de Sgt. Pepper Lonely Hearts Club Band. Le chant est exprimé visuellement par des bulles oblongues et agrémentées, qui circulent d'une case à l'autre avec les paroles de la chanson; la police de caractères utilisée est manuscrite et cotonneuse. Ici et là, on peut voir des notes musicales fluctuer dans l'air, donnant idée du fait que la musique remplit tout le Ciel et convoque les dieux, qui viennent joyeusement participer au chant.

Une stratégie similaire apparaît dans la série mensuelle italienne Lazarus Ledd (1992-2015). Dans le premier numéro (CAPONE; OLIVARES, 1993), le héros conduit son taxi durant une nuit pluvieuse à New York. L'homme écoute l'autoradio, qui diffuse One (1991) du groupe de rock U2. Nous, en tant que lecteurs, remarquons plusieurs détails de ce qui se passe au niveau auditif: 1. lorsqu'un client monte dans la voiture ( $1{ }^{\text {ère }}$ case), Lazarus démarre le taximètre (tlak, $2^{\text {ème }}$ case) et allume la radio (click, $3^{\text {ème }}$ case); 2 . la voix d'un animateur sort de la radio ( $4^{\text {ème }}$ case) et on peut voir que la bulle de textes associée est différente de la voix du client ( $1^{\text {ère }}$ case): dans ce cas, on comprend au contour de la bulle que le son provient d'une source artificielle et nous sommes amenés à imaginer la voix accompagnée du crépitement des sons provenant des appareils radio; 3. dans les trois dernières cases, les seuls sons visualisés sont les paroles 
de One, émises par la radio et exprimées avec une inscription manuscrite. Le lecteur est censé connaître cette chanson et donc (espérons-le, du point de vue des auteurs) la chantera dans sa tête, donnant une ambiance à toute la scène qui correspond au ton douloureux de la pièce; 4. il existe plusieurs indicia qui nous incitent à imaginer des sons supplémentaires: le martèlement de la pluie, le bruit des essuie-glaces, peut-être les bruits venant de la route - moteurs, voitures klaxonnantes etc.; 5. enfin, un détail intéressant est qu'au début de cet album de BD, l'auteur présente une note dans laquelle il encourage les lecteurs à apprécier l'histoire tout en écoutant l'album Achtung Baby de U2. Nous avons ici un croisement entre la musique intra-diégétique et la musique extra-diégétique, destiné à favoriser le plaisir auditif.

Figure 15 - Ade Capone et Giancarlo Olivares, Lazarus Ledd, n. 1 (1993), page 8 (tableau 4). Avec l'aimable autorisation de Giancarlo Olivares et des Heirs d'Ade Capone/Giancarlo Olivares. Lazarus Ledd ${ }^{\circledR}$ et ${ }^{\mathrm{TM}}$ Heirs Ade Capone. Lazarus Ledd n. $1^{\odot}$ Heirs Ade Capone/Giancarlo Olivares
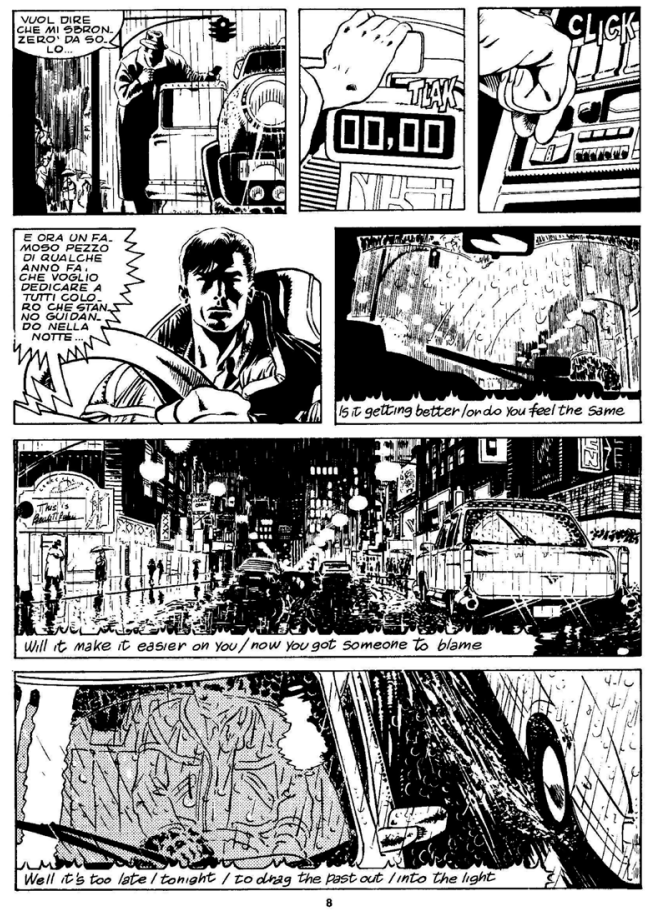

En guise de premier exemple de la deuxième stratégie, prenons l'histoire de six pages El Mago (1982) des créateurs argentins Carlos Trillo et Domingo Mandrafina ${ }^{13}$. C'est une $\mathrm{BD}$ sans bulles, dans laquelle un prestidigitateur exécute un numéro avec l'aide d'une belle assistante. Une musique suave accompagne le numéro. Comment savons-nous qu'il y a de la musique et qu'elle est suave? Le lecteur est conscient que de la musique accompagne l'action sur scène à partir

13 Les lecteurs peuvent admirer cette histoire au lien suivant: http://letteraturagrafica.over-blog.com/article-protofumetti-di-oggi-carlos-trillo-mandrafina-il-mago-da-l-eternauta-n-1- marzo-1982-89675099.html. 
des notes dessinées à l'intérieur des cases; et l'idée que la musique est suave est déduite des mouvements de l'assistante, qui se glisse de façon surprenante dans le chapeau du magicien et, à partir de là, exécute une sorte de strip-tease en enlevant de façon lascive ses bas, son soutien-gorge et sa culotte.

Comme deuxième exemple de la seconde stratégie, à comparer directement avec la précédente, considérons une page du premier numéro de ESP (19951999), une autre série de BD italiennes. Dans El Mago, nous pouvions voir des cœurs symboliques fluctuant dans la case et contenant de petites notes de musique, afin de suggérer que la musique était dans la case, proche du magicien; dans la page de cet épisode de ESP (LA NEVE; CARACUZZO, 1995), le personnage à l'œuvre est plutôt en train d'écouter une musique qui vient d'ailleurs: elle s'écoule depuis une ambiance cachée, de pièces, de couloirs, jusqu'aux oreilles du personnage. C'est une musique sans mots et nous ne pouvions pas connaître le type exact de musique si dans la première case un texte de légende (faisant allusion aux pensées du personnage à l'œuvre) n'avait pas fait nous savoir que cette musique est un "air déchirant ". Dans la dernière case, nous découvrons que le chant vient d'une petite fille allongée sur un lit. De cette façon, le lecteur est amené à recadrer rétrospectivement toute la scène et à ré-imaginer la chanson avec la voix sans paroles, d'un enfant qui fredonne. En ce sens, le besoin d'une expérience de lecture complète, renforcée par ces éléments auditifs, pourrait amener certains lecteurs à relire la séquence, en utilisant les informations supplémentaires acquises lors de la première lecture.

Figure 16 - Michelangelo La Neve et Giancarlo Caracuzzo, ESP, n. 1, 1995, p. 91. Avec l'aimable autorisation de Michelangelo La Neve et Giancarlo Caracuzzo. $\mathrm{ESP}^{\odot} 1995$ Michelangelo La Neve/Giancarlo Caracuzzo

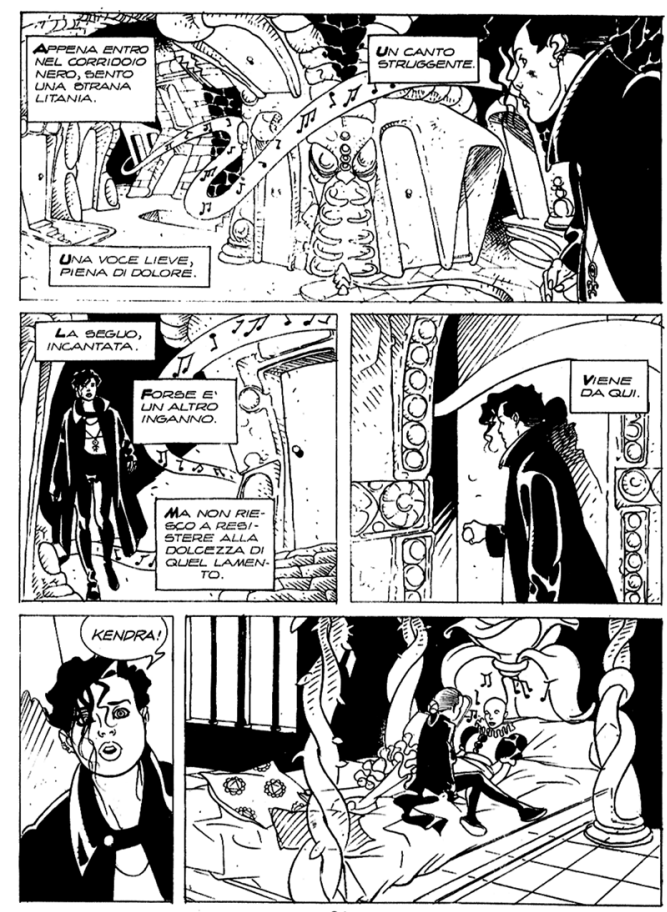




\section{SUGGÉRER LE SON: ALLUSION VISUELLE ET ILLUSION AUDITIVE}

Comment est-il possible d'entendre réellement quelque chose émanant des $\mathrm{BD}$ ? Si l'on exclut la notion de BD en tant qu'objet physique (tel qu'analysé dans HAGUE, 2013, susmentionné), on se limite à considérer ce que l'on voit sur la page imprimée. Nous rencontrons ici l'un des nombreux paradoxes de la communication narrative et multi-planaire des BD et nous pouvons remarquer une différence importante avec un médium voisin, l'illustration. Dans toute illustration typique, la scène est généralement " muette ", quand bien même elle représente une situation très vivante, car le dessin est censé décrire et représenter quelque chose de vivant, comme cela se produit dans les photographies, qui constituent un " passé re-présenté " (FERRAROTTI, 1992, p. 8). Au contraire, les BD regorgent souvent de textes verbaux, de lignes cinétiques, d'onomatopées et d'autres éléments contribuant à faire sentir au lecteur que chaque case de la page est fonction de deux variables: l'espace et le temps.

Même dans une illustration explicitement destinée à exprimer une situation vivante, les voix, sons et bruits supposés provenant des personnages et des objets de la scène " appartiennent " à l'image autant que les autres variables, telles que les couleurs, la luminosité de l'image et les silhouettes et objets. Tout a, avant tout, un accent descriptif plutôt que narratif (qui est présent, mais secondaire): un exemple frappant est Homecoming G.I., une célèbre illustration de 1945 de Norman Rockwell (cf. KNIGHT, 2013). Au contraire, dans une case de BD ou dans un groupe de cases dans lesquelles on voit deux personnes parler ou un océan houleux, même en l'absence de bulles ou d'onomatopées ce que nous voyons n'est pas seulement une scène descriptive, mais aussi, et surtout, une narration pure: les sons sortent en théorie de la page car ils sont rappelés par la manière dont la scène est construite graphiquement. Je peux ainsi introduire la notion de dessin soustractif: les non-signes visuels (c'est-à-dire leur absence totale) qui sont aussi efficaces que les signes effectivement dessinés, mais qui, d'une certaine manière, s'avèrent plus forts par leur rareté dans un médium généralement riche en signes symboliques ajoutés aux dessins illustratifs pour indiquer la présence de voix et de sons.

Ce jeu subtil entre la présence et l'absence explicite ou implicite de mots, de sons et de bruits dans les $\mathrm{BD}$, et plus particulièrement dans le cas de la présence/absence du mot parlé, a été commenté de manière sensible par Daniele Barbieri (1996, p. 16):

Toutes les bandes dessinées sont construites comme une relation entre des images et des mots, même celles dans lesquelles il n'y a pas de mots, car leur absence, dans un contexte où ils sont généralement présents, est très significative. Une erreur répandue est de croire que, puisque les valeurs représentatives de la $B D$ ne sont généralement pas comparables à celles des arts visuels, et que les valeurs littéraires de la BD ne sont pas comparables à celles du roman, la BD ne peut pas espérer atteindre le niveau de considération attribué à l'un ou à l'autre: beaucoup oublient que dans la BD la vraie valeur tient dans la relation entre les mots et les images, et non dans le premier ou le second pris de manière séparée. Et cette relation se dévoile de multiples façons, difficiles à cataloguer.

La relation entre silence et bruit se produit également lorsqu'une complicité culturelle et émotionnelle s'établit entre le lecteur et le récit. Les auteurs devraient parvenir à faire entrer le lecteur dans un état de félicité littéraire; un " 
abandon " au flux de la narration. Mais le lecteur devrait également être disposé à participer à ce processus interne qui passe également par certaines conditions de lecture, telles que le calme, la concentration, l'atmosphère et le confort.

Dans une page du deuxième numéro (VIETTI; OLIVARES, 1995) de la série de science-fiction Hammer (1994-1996), un module spatial se déplace d'une station en orbite à un troisième engin. Sur le plan visuel, le lecteur remarque tout de suite la verticalité de la disposition, la technologie avancée des engins et les flammes de propulsion de la navette. Nous remarquons également les ombres sur les objets qui nous indiquent à quel point le contraste entre lumière et obscurité est net dans l'espace extra-atmosphérique; la trajectoire de la navette de la station vers l'autre engin; le mouvement de rotation de la station d'une case à l'autre; et notre point de vue en tant que lecteurs s'éloignant de la station. Toute la séquence est un hommage à 2001: Odyssée de l'Espace (1968) de Stanley Kubrick, le film qui a modifié pour toujours, entre autres, le paysage sonore du cinéma de science-fiction grâce au réalisme de ses scènes dans l'espace. Dans cette page également, nous ne pouvons ni voir ni entendre les sons, car dans l'espace extra-atmosphérique le son ne peut pas se propager. Mais dans le même temps, les lecteurs qui partagent avec les auteurs de Hammer les codes du cinéma de science-fiction peuvent ajouter des effets sonores à leur propre reconstitution mentale de la scène, en recourant à des sons typiques de ce paysage sonore cinématographique: des notes monotones d'un hautbois ou d'un cor, le sifflement du jet enflammé de la navette etc.

Figure 17 - Stefano Vietti et Giancarlo Olivares, Hammer, n. 2, "Doppia fuga " ('Double fuite'), Juillet 1995, Edizioni Star Comics, p. 2. Avec l'aimable autorisation de Stefano Vietti et Giancarlo Olivares. Hammer n. $2^{\odot}$ Stefano Vietti/Giancarlo Olivares
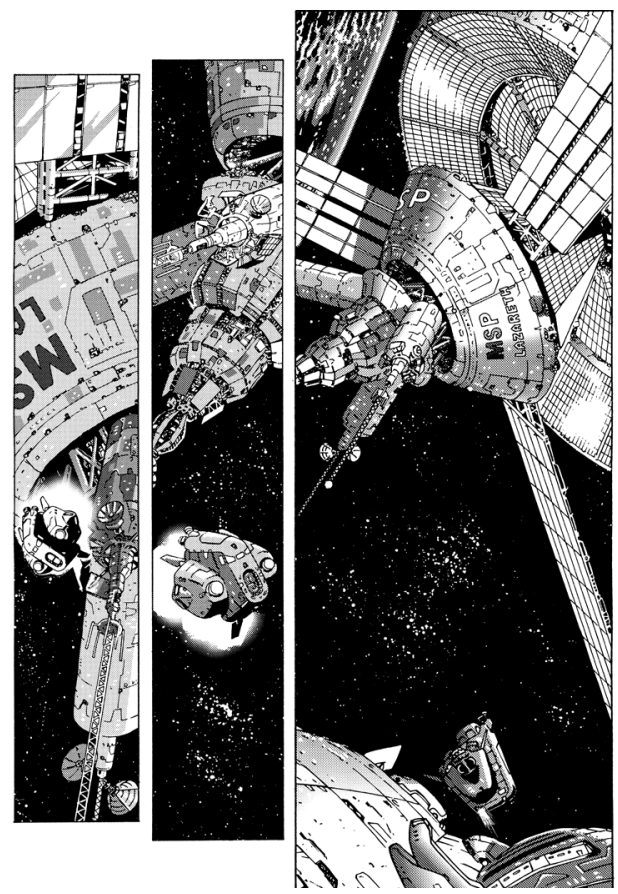
L'influence du cinéma de science-fiction sur la suggestion de sons ou de silence dans les BD est répandue: dans une histoire de Katsuhiro Ōtomo (1980), une scène surréaliste inhabituelle rend à nouveau hommage à Kubrick: deux astronautes pénètrent dans un vaisseau spatial apparemment abandonné, à la dérive dans l'espace profond, et découvrent que ses intérieurs sont entièrement meublés comme dans une maison britannique du début du $\mathrm{XX}^{\mathrm{e}}$ siècle. Les multiples contrastes entre le vaisseau spatial et ses intérieurs très terrestres, et entre ces meubles de style édouardien et les astronautes futuristes et entièrement équipés, donnent au lecteur le sentiment d'être désorienté mais, dans le même temps, ils fournissent aussi des indices sur l'ambiance sonore de la scène: la pièce baigne dans le silence total mais, encore une fois, à travers une inter-textualité et une pré-textualité avec le cinéma (ECO, 1984b), nous sommes censés entendre au moins les heurts légers des bottes des scaphandres sur le tapis et les souffles à l'intérieur des casques.

\section{Conclusion}

Dans ces pages, j'ai essayé de montrer les implications d'une dimension auditive des BD. Certes, la recherche sur ce sujet complexe n'est pas achevée et elle implique une approche multidisciplinaire. En guise de conclusion et pour tenter de relier l'objectivité de la lecture d'une BD à la subjectivité des sentiments et aux suggestions sensorielles que les lecteurs proactifs peuvent convoquer pour un plaisir plus intense, je rappellerai au lecteur l'ancienne notion philosophique d'Erlebnis, une " expérience vivante " ou " expérience vécue ", par procuration, supposée complète et vivante: ses perceptions personnelles, ses expériences et aussi le contenu même de sa propre conscience sont reçus ou formulés non pas de manière passive mais, au contraire, capturés et extrêmement bien compris au travers du flux intérieur de la conscience. Alors que les implications philosophiques d'Erlebnis s'étendent à la compréhension de l'histoire, des logiques et des lois de la pensée, je me réfère ici à Erlebnis tel qu'il a été défini par Edmund Husserl (1973 [1900-1901]) dans ses Logische Untersuchungen, une " conscience intentionnelle ", concept qui remonte à Aristote sous le nom de noesis, l'acte de concevoir des images et des idées dans son propre esprit à travers l'action même de la pensée.

L'activité mentale impliquée dans la lecture de BD et dans la construction interne de déclencheurs sensoriels simulés et imaginés (y compris, et en particulier, l'imagination auditive, tout à fait remarquable dans un médium imprimé de façon simple et maigre sur papier), est la fusion d'un acte de bonne volonté et d'un acte d'abandon intellectuel aux signaux que la bande dessinée tente de nous envoyer à travers ses deux puissants outils: les procédés graphiques et la narration verbo-visuelle.

\section{SOUND IN COMIC ART: REPRESENTATION AND IMAGINATION OF THE AURAL DIMENSION}

Abstract: Hearing is the second more important physiological sense brought up when creating and then reading comics. Comics display, or may display, visual signs and symbols that semiotically refer to sounds and noises. This essay is a general outlook of the ways the visualisation of sounds can occur 
in comics narratives. It discusses the main features of how comics convey sounds and how the combined action of visual signs and effective story-telling can encourage readers's sonic imagination to create or recreate in their minds personal versions of sounds.

Keywords: Sound. Onomatopoeias. Lettering.

\section{BIBLIOGRAPHIE}

BARBIERI, D. I linguaggi del fumetto. Milan: Bompiani, 1991.

BARBIERI, D. Alchimie tra immagini e parole. Domenica (supplément de dimanche de Il Sole -24 Ore, quotidien), 4 fevrier, p. 16, 1996.

BAUDELAIRE, C. OEuvres complètes de Charles Baudelaire: curiosités esthétiques. Paris: Michel Lévy Frères, 1868.

BECKER, S. Comic art in America: a social history of the funnies, the political cartoons, magazine humor, sporting cartoons and animated cartoons. New York: Simon \& Schuster, 1959.

BERARDI, G.; MILAZZO, I. Ken Parker 36. " Diritto e rovescio ". Janvier-fevrier. Milan: CEPIM, 1981.

BERBERIAN, C. Stripsody. Solo voice. New York: C. F. Peters, 1967.

BERONÄ, D. Wordless books: the original graphic novels. New York: Harry N. Abrams, 2008.

BRINGHURST, R. The elements of typographic style. Point Roberts, WA: Hartley \& Marks, 1992.

BUSCH, W. Der Virtuos. Ein Neujahrsconcert. Supplement à Fliegende Blätter, 1865. v. XLIII.

CAPONE, A.; OlIVARES, G. Lazarus Ledd 1. " Doppia identità ". Juillet. Perugia: Star Comics, 1993.

CARMI, E. Stripsody. Interprétation vocale de Cathy Berberian. Introduction d'Umberto Eco. Rome: Arco d'Alibert; Houston: Kiko Galleries, 1966.

CASTELLI, A. Eccoci ancora qui! 1895-1919: i primi 25 anni del fumetto americano per quotidiani. Milan: Edizioni IF, Museo Nazionale del Fumetto, 2006.

CHIAREllo, M.; KLEIN, T. The DC Comics Guide to Coloring and Lettering. New York: Watson-Guptill, 2004.

COOK, R. T. Why comics are not films: meta-comics and medium-specific conventions. In: MESKIN, A.; COOK, R. T. (org.). The art of comics: a philosophical approach. Chichester: Wiley-Blackwell, 2012. p. 165-187.

$\mathrm{COHN}, \mathrm{N}$. The visual language of comics: introduction to the structure and cognition of sequential images. London: Bloomsbury, 2013.

COUPERIE, P. A history of the comic strip. New York: Crown Publishers, 1968.

COVEY, S. Beyond the balloon: sound effects et background text. In: JOHNSON, L. For better or for worse. ImageText: interdisciplinary comics studies 2. 2006. 
CREPAX, G. Valentina. 78 histoires en éditions diverses. Milan: Milano Libri Mondadori, 1965-1995. De la NÉZIĖRE, R. La leçon dans le phonographe. Mon Journal, n. 26, 26 mars 1904.

DETTI, E. Il fumetto tra cultura e scuola. Scandicci, FI: La Nuova Italia, 1984. DORÉ, G. Des-agréments d'un voyage d'agrément. Paris: Aubert et Cie, 1851. Nouvelle édition: Strasbourg: Éditions 2024, 2013.

DROOKER, E. Flood! A novel in pictures. Milwaukie: Dark Horse, 1992.

ECO, U. Lettura di Steve Canyon. Comunicazioni di massa 2, mars-juin 1964, p. 4-5 et 41-76. Rome: Centro di Sociologia delle Comunicazioni di Massa - Istituto di Pedagogia dell'Università di Roma.

ECO, U. Semiotics and the Philosophy of Language. Bloomington: Indiana University Press, 1984a.

ECO, U. Tipologia della ripetizione. In: L'immagine al plurale. Serialità e ripetizione nel cinema e nella televisione. Dirigé par F. Casetti. Venice: Marsilio, 1984b. p. 19-35.

EISNER, W. Comics \& sequential art. Tamarac, FL: Poorhouse Press, 1985.

FERRAROTTI, F. Mass media e società di massa. Bari: Laterza, 1992.

FORCEVILLE, C. Creative visual duality in comics balloons. In: VEALE, T.; FEYAERTS, K.; FORCEVILLE, C. Creativity and the agile mind: a multi-disciplinary study of a multi-faceted phenomenon. Berlin; Boston: Walter de Gruyter, 2013. p. 253-270.

FORCEVILLE, C.; VEALE, T.; FEYAERTS, K. Balloonics: the visuals of balloons in comics. In: GOGGIN, J.; HASSLER-FOREST, D. The rise and reason of comics and graphic literature: critical essays on the form. Jefferson: McFarland, 2010. p. 56-73.

FRANCESCHI, L. Le interiezioni primarie nei fumetti italiani contemporanei. Memoire. Pisa: Université de Pisa, 2009.

GADDUCCI, F. Notes on the early decades of Italian comic art. Pisa: Felici, 2006. GADDUCCI, F.; TAVOSANIS, M. La scrittura non standard nei fumetti italiani. In: PETRINI, D. Die Sprache(n) der Comics. Kolloquium in Heidelberg, 16.-17. Juni 2009. Munich: Martin Meidenbauer, 2009. p. 113-126.

GARBUGLIA, A. Stripsody. La vocazione musicale delle strisce a fumetti. Macerata: Edizioni Universitarie di Macerata, 2011.

GRANDVILLE, J.-J. Une Valse. Le Magasin pittoresque. Dirigé par Édouard Charton. Paris, 1840. v. vIII, p. 244-245.

GUILLAUME, A. Les perfidies du téléphone. Le Journal pour tous, 3 jan. 1894.

GUYNES, S. A. Four-color sound: a perceian semiotics of comic book onomoatopoeias. Public Journal of Semiotics, v. 6, n. 1, p. 58-72, 2014.

HAGUE, I. Beyond the visual: the roles of the senses in contemporary comics. Scandinavian Journal of comic Art, v. 1, n. 1, p. 98-110, 2012.

HAGUE, I. Comics et the senses: a multisensory approach to comics and graphic novels. New York; London: Routledge, 2013.

HATFIELD, C. Alternative comics: an emerging literature. Jackson: University Press of Mississippi, 2005. 
HUSSERL, E. Logical investigations. Dirigé et traduit par J. N. Findlay. Londres: Routledge, 1973.

IHDE, D. Auditory imagination. In: BULL, M.; BACK, L. The auditory culture reader. Oxford; New York: Berg, 2003. p. 61-66.

IMAGE [\&] NARRATIVE. Special issue: The Narrative Functions of Photography in Comics, v. 16, n. 2, 2015.

IRVING, C. Chris Ware on building a better comic book. Graphic NYC, 6 mars 2012. KATSURA, M. Den'ei shōjo Video Girl Ai. Weekly Shōnen Jump. Tokyo: Shūeisha, 1990-1993.

KNIGHT, M. K. Redheads Rule!. The Saturday Evening Post, mai-juin 2013.

KHORDOC, C. The comic book's soundtrack: visual sound effects in Asterix. In: VARNUM, R.; GIBBONS, C. T. The language of comics: word et image. Jackson, MS: University Press of Mississippi, 2001. p. 156-173.

KUNZLE, D. The early comic strip: narrative strips and picture stories in the European broadsheet from c. 1450 to 1825. Berkeley: University of California Press, 1973.

KUPER, P. Franz Kafka's The Metamorphosis adapted by Peter Kuper. Danvers: Crown, 2003.

LACASSIN, F. The comics strip and film language. Traduit par D. Kunzle. Film Quarterly, v. 26, n. 1, p. 26-33, 1972.

LA NEVE, M.; CARACUZZO, G. Esp, \#1, décembre 1995. "Il giardino delle anime ". Rome: Universo Pubblicità, 1995.

LEFÈVRE, P. Some medium-specific qualities of graphic sequences. SubStance, v. 40, n. 1, p. 14-33, 2011.

LEONE, C. Delitto al Cineclub. Corto Maltese, vol. viII, n. 6, juin 1990.

LINDEKENS, R. Analyse structurale de la Stripsody de Cathy Berberian. Communications, n. 24, p. 140-176, 1976.

MANARA, M. John Lennon. In: MANARA, M. Storie brevi. Rome: Nuova Frontiera, 1993.

MASEREEL, F. 25 images de la passion d'un homme. Genève: Éditions du Sablier, 1919.

MASSARUTTO, F. Assoli di china. Fra jazz e fumetto. Rome: Stampa Alternativa, 2011.

MCCLOUD, S. Understanding comics: the invisible art. Amherst: Kitchen Sink Press, 1993.

MCCLOUD, S. Reinventing comics: how imagination and technology are revolutionizing an art form. New York: Paradox Press, 2000.

MCCLOUD, S. Making comics: storytelling secrets of comics, manga and graphic novels. New York: Harper Paperbacks, 2006.

MCKEAN, D. Cages. 10 issues. Northampton: Tundra Publishing; Princeton: Kitchen Sink Press, 1990-1996.

MILLER, F. Sin city. Dans Dark Horse Presents, n. 51-62 (mai 1991-juin 1992). Milwaukie: Dark Horse, 1991-1992. 
NENCIONI, G. Avant-propos pour L. Becciu. Il fumetto in Italia. Florence: Sansoni, 1971.

ŌTOMO, K. Kanojo no omoide. Young Magazine, Tokyo, n. 17, nov. 1980.

OTT, T. Exit. Paris: Delcourt, 1997.

PELLITTERI, M. Sense of comics. La grafica dei cinque sensi nel fumetto. Rome: Castelvecchi, 1998.

PELLITTERI, M. Fumetto, apripista della multimedialità. I tre piani della percezione nei fumetti. Liber, n. 43, p. 38-44, juillet-septembre, 1999.

PELLITTERI, M. Pornography and sinaesthesia in Manga: multi-sensorial reception of Eros in Japanese comics. International Journal of Comic Art, v. 9, n. 2, p. 425-440, 2007.

PELLITTERI, M. Lettering in comics: giving sound to a silent medium. Dans Critical Survey on Graphic Novels: History, Theme, et Technique, dirigé par B. Beaty et S. Weiner. Ipswich: EBsco, 2012. p. 263-265.

PELLITTERI, M. Die fünf Sinne und Synästhesie in Comics. Annäherende Erklärungsversuche über Wie und Warum Comiclesen nicht nur den Sinn des Sehens betrifft. Dans Erzählen im Comic, dirigé par O. Brunken. Bochum: Bachmann, 2013. p. 49-59.

PETERSEN, R. S. The acoustics of Manga. In: HEER, J.; WORCESTER, K. A comics studies reader. Jackson: University of Mississippi Press, 2009. p. 163-171.

PIETRINI, D. Parola di papero. Storia e tecniche della lingua dei fumetti Disney. Florence: Cesati, 2009.

POLLMANN, J. Shaping sounds in comics. International Journal of Comic Art, v. 3, n. 1, p. 9-21, 2001.

POSOCCO, C. MangArt: forme estetiche e linguaggio del fumetto giapponese. Milan: Costa \& Nolan, 2005.

RABIER, B. Le phono-piège. In: RABIER, B. Les cent bon tours. Paris: Tallandier, 1907.

RIES, H. et al. Wilhelm Busch: Die Bildergeschichten. Historisch-kritische Gesamtausgabe in drei Bänden. Schlütersche Buchhandlung: Hannover, 2007. 3 tomes.

SABIN, R. The crisis in Modern American et British comics, and the possibilities of the internet as a solution. In: MAGNUSSEN, A.; CHRISTIANSEN, H.-C. Comics et culture: theoretical approaches to reading comics. Copenhagen: Museum Tusculanum Press, 2000. p. 43-57.

SAMPAYO, C.; MUÑOZ, J. Billie Holiday. Milan: Rizzoli, 1993.

SANTIROSI, S. Craig Thompson: "I fumetti sono spartiti musicali ". L'Unità, 4 janvier 2012.

SAUSVERD, A. "La leçon dans le phonographe " par Raymond de la Nézière. Töpfferiana, 14 janvier 2009a.

SAUSVERD, A. (2009b). 'Le phono-piège' par Benjamin Rabier. Töpfferiana, 9 fevrier 2009b.

SMOLDEREN, T. Ceci n'est pas une bulle! - structures énonciatives du phylactère. RHRT, 31 août 2006. 
STARKINGS, R.; ROSHELL, J. "JG ". Comic book lettering: the comicraft way. Los Angeles: Active Images, 2003.

TAN, S. The arrival. New York: Arthur A. Levine Books, 2007.

THOMAS, M. The invisible art in plain sight: a look at the art of lettering. Comic Book Resources, 9 juin 2000.

TINSLEY, K. Digital prepress for comic books: the definitive desktop production guide. New York: Stickman Graphics, 1999.

TORELLI LANDINI, E. Grafia musicale e segno pittorico nell'avanguardia italiana, 1950-1970. Rome: De Luca, 2012.

TOSTI, A. Graphic Novel. Storia e teoria del romanzo a fumetti e del rapporto fra parola e imagine. Latina: Tunué, 2016.

TRILLO, C.; MANDRAFINA, D. El mago (1982). In: TRILLO, C.; MANDRAFINA, D. Senza parole. Rome: ACME, 1991. (Sèrie "Blackbirds").

VIETTI, S.; OlIVARES, G. Hammer n. 2. "La caccia ". Juillet. Perugia: Star Comics, 1995.

WAGNER, R. Die Kunst und die Revolution. Leipzig: Otto Wigand Verlag, 1849. ZAMARIN, R. Stripsody. Solo voice. New York: C. F. Peters, 1967.

Recebido em $1^{\circ}$ de outubro de 2018. Aprovado em 23 de março de 2019. 